\title{
Les échelles de la recherche et du projet
}

The Scales of Research and Project

\section{Bernardo Secchi}

Traducteur : Anne Grillet-Aubert

\section{(2) OpenEdition}

\section{Journals}

Édition électronique

URL : http://journals.openedition.org/crau/316

DOI : $10.4000 /$ crau.316

ISSN : 2547-5746

\section{Éditeur}

Éditions du patrimoine

Édition imprimée

Date de publication : 1 décembre 2009

Pagination : 175-201

ISBN : 978-2-85822-944-4

ISSN : 1296-4077

\section{Référence électronique}

Bernardo Secchi, «Les échelles de la recherche et du projet », Les Cahiers de la recherche architecturale et urbaine [En ligne], 24/25 | 2009, mis en ligne le 01 septembre 2017, consulté le 01 mai 2019. URL: http://journals.openedition.org/crau/316 ; DOI : 10.4000/crau.316 
À la demande du Comité d'orientation des Cahiers, Bernardo Secchi avait élaboré avec Corinne Tiry le projet d'un dossier consacré à "La grande échelle ». C'était en 2007 et la Consultation internationale sur « Le grand pari de l'agglomération parisienne " était à peine lancée. Bernardo Secchi a élaboré une candidature pour participer à cette opération. Son équipe a été sélectionnée parmi les dix équipes pluridisciplinaires retenues. Mais, pour des raisons déontologiques évidentes, la rédaction a jugé préférable de ne pas donner suite à la publication de ce dossier. La présente livraison propose cependant la contribution généreuse que Secchi avait fournie à l'époque. Avec ce qu'il faut d'engagements théoriques et de convictions doctrinales, elle éclaire d'un jour nouveau les relations critiques que l'aménagement de l'espace entretient avec «le savant et le politique ».

\section{Les échelles de la recherche et du projet}

BERNARDOSECCHI

De manière générale, il y a de bonnes raisons pour vouloir réfléchir à nouveau aux différentes échelles de la recherche et du projet concernant la ville et le territoire. Il y a, en particulier, de bonnes raisons pour vouloir réfléchir à nouveau à la question de la grande échelle, traitée à plusieurs reprises au cours du XX $X^{e}$ siècle avec des réponses à chaque fois différentes.

Les urbanistes et les architectes, les géographes et les historiens, les sociologues et les économistes, les administrateurs et les politiques, tous ont vu dans la grande échelle la possibilité de mieux comprendre la ville (avec ses rythmes et ses formes de croissance) et de mieux appréhender les relations entre la ville, la campagne et les territoires distants les uns des autres. Entre une approche «mercantiliste » et une approche "physiocratique », tous ont perçu en elles l'opportunité de comprendre les articulations entre l'urbanisation, l'économie et le paysage, afin de mieux les organiser et mieux les « dessiner ». Dans le même temps, ils ont appréhendé l'échelle du local, plus réduite et plus limitée, comme une possibilité de saisir au plus près la spécificité de chaque lieu, de chaque situation, demande, comportement ou problème. 
C'est pour cela qu'ils ont constamment cherché à approfondir et à élargir le cadre de leurs recherches, à sortir des limites académiques. Ils ont toujours voulu s'extraire des hiérarchies administratives et institutionnelles qui les contraignaient dans des échelles spatio-temporelles généralement sans lien avec la réalité des transformations de la ville et du territoire et sans rapport avec les questions urgentes posées par les politiques urbaines et territoriales ou les projets d'urbanisme et d'architecture. II s'agit là d'une histoire bien connue.

Aujourd'hui, les raisons qui poussent à la réflexion sur les échelles de la recherche et du projet sont en partie différentes, tout au moins en Europe continentale. II me semble utile de les exposer sous la forme de « thèses » qui doivent pouvoir être discutées, contredites, articulées, modifiées sur la base des expériences de ces dernières années.

De façon très synthétique, les thèses que je soutiens peuvent s'exprimer de la manière suivante. Pendant quelques décennies, l'approche prédominante a été celle de la description, de l'interprétation et du projet urbain, c'est-à-dire une approche bottom up qui a privilégié les dimensions et les caractéristiques de chaque lieu tout en refusant les stratégies traditionnelles comprehensive. Or, plusieurs des principaux scénarios auxquels nous serons inévitablement confrontés dans un futur proche nous conduisent désormais à réhabiliter une approche opposée, de type top down, et à parcourir systématiquement dans les deux sens, non seulement les échelles spatiales, mais aussi les échelles identitaires et leur histoire, les échelles du pouvoir et de ses différentes formes, les échelles du public et du privé avec leurs glissements réciproques, les échelles du politique et du projet.

\section{Bottom up}

Les dernières décennies ont été marquées dans le monde entier, pas seulement en Europe, par un vaste réexamen des problèmes de la ville et du territoire «à partir du bas », c'est-à-dire à partir de l'observation, de la description et de l'interprétation de la diversité des comportements quotidiens, individuels et collectifs ${ }^{1}$. Cette phase, importante, a démontré l'intérêt d'une approche de la recherche dite by design, c'est-à-dire la valeur du projet comme outil fondamental de construction des connaissances, et non pas seulement comme simple vecteur d'application des connaissances. Ces recherches ont déconstruit la logique qui liait traditionnellement les différents " matériaux élémentaires ${ }^{2}$ " de l'interprétation et du projet de la ville et du territoire ${ }^{3}$. Isolé et analysé en soi, chacun de ces matériaux devenait ainsi disponible pour la construction de nouvelles compositions, agrégations et juxtapositions, pour la construction de séquences finalement toutes différentes de celles qu'avait pu mettre en œuvre la modernité au cours d'un long travail d'accumulation mais qui, une fois dépassées, apparaissaient totalement incongrues (Viganò, 1999) ${ }^{4}$.

Pour les urbanistes et les architectes, notamment, les tentatives pour saisir le sens de ces nouvelles compositions, agrégations et juxtapositions ont suscité l'urgence d'une redéfinition de leur rôle dans la perception des dimensions matérielles et immatérielles du welfare. Cette urgence est née à un moment où, après l'abandon de toutes les visions holistiques, la société n'était plus considérée comme un tout cohérent formé de parties complémentaires, à un moment où le territoire n'était plus représenté par la dichotomie ville/campagne, où la ville
1. Il est évidemment difficile de donner les dates de début et de fin de chaque période, mais je ne m'éloigne probablement pas trop de la vérité en disant que le mouvement auquel je me réfère commence, malgré d'inévitables anticipations ou retards selon les pays, entre les années 1960 et 1970. Aujourd'hui, avec le recul, on peut comprendre les raisons de ce déplacement de point de vue, commun d'ailleurs à beaucoup de disciplines, à l'économie comme à la sociologie ou à l'histoire. Les schémas conceptuels avec lesquels on avait l'habitude d'observer la ville et le territoire, de les dessiner et de chercher à gouverner leurs principales transformations étaient devenus une sorte de bon sens et de savoir commun des experts et des administrateurs, mais ils se sont révélés, au début des années 1970, inefficaces sur le plan analytique comme sur le plan des constructions politiques et du projet. Quelque chose ne fonctionnait plus. Toutes les disciplines ont alors mesuré la nécessité de revenir à une description minutieuse de leur propre terrain d'étude par une approche micro, qui n'impliquait rien alors à l'égard des grands cadres de cohérence macro. Une opération qui a cependant mis en évidence de nombreux problèmes nouveaux, difficilement analysables dans les schémas traditionnels. Voir Alessandro Pizzorno, Il velo della diversità. Studi su razionalità e riconoscimento, Milan, Feltrinelli, 2007. 


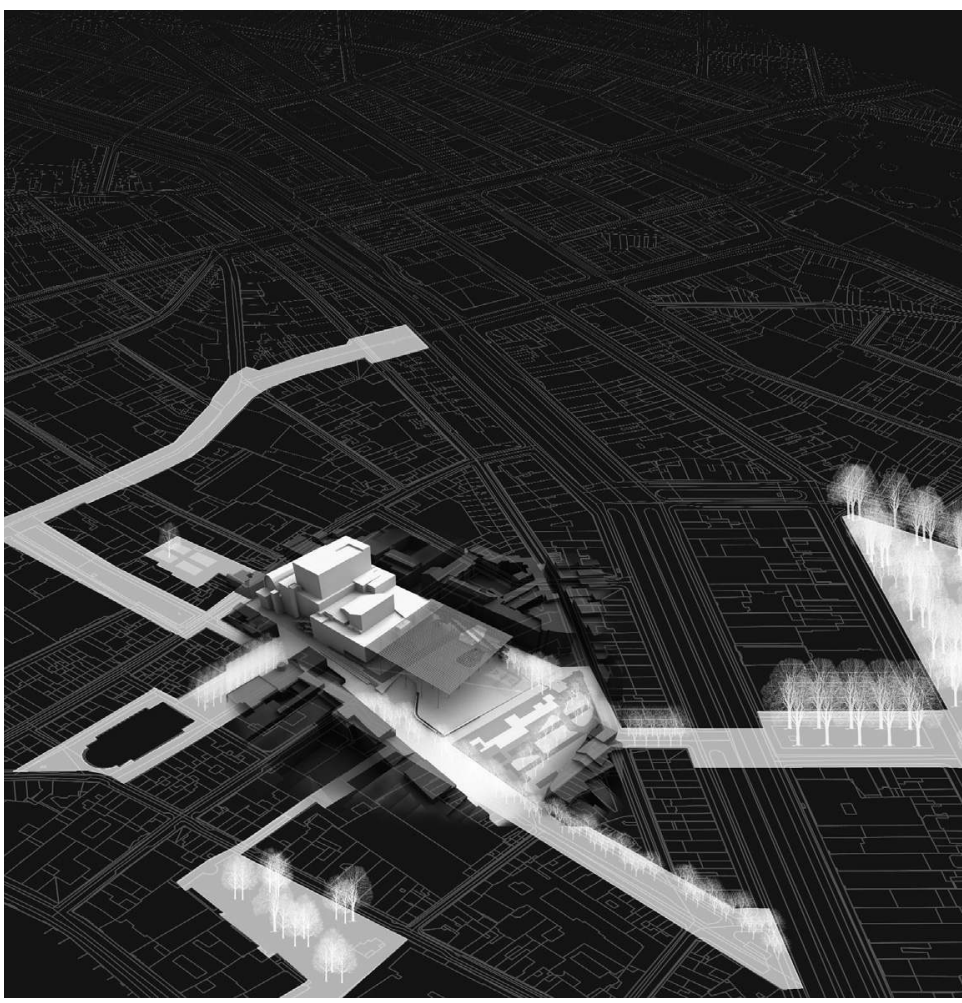

Anvers (Belgique).

Vue d'ensemble

de la place des théâtres

et de ses connections

à la ville.
DEUX PARCOURS D'ANALYSE

«Dans tous les plans et les projet de Studio Secchi-Viganò, nous avons suivi en même temps deux parcours $d$ 'analyse et de projet, de connaissance de la ville et du territoire :

- un parcours top-down qui part des éléments de la géographie, des réseaux hydrauliques, de la topographie du paysage et des grandes infrastructures. Ce parcours amène à une compréhension du territoire et de sa structure spatiale, ce qui devient le point de départ de tout projet.

- un deuxième parcours qui part d'une interrogation des habitants et des lieux et qui essaie de découvrir leurs attentes et leurs imaginaires, leurs spécificités et leurs inerties. Tout projet doit se confronter avec I'inertie qui est la véritable mesure du temps: inertie des objets physiques et inertie des idées.

Le projet d'un territoire, d'une ville telle que la métropole anversoise ou le territoire de la province de Pescara ou de la ville de Pesaro, tout projet d'un lieu spécifique tel que le parc Spoornoord ou le Theaterplein à Anvers doit essayer d'interpréter les opportunités offertes par le territoire ou les lieux que les habitants, avec leurs imaginaires, peuvent accueillir. " B. SECCHI

(C) Illustrations Secchi-Viganò

4. P. Viganò, La Città elementare, Milan, Skira, 1999.

\section{Pour préciser ce que j'entends par} " matériau élémentaire », je ne vois rien de mieux que la belle citation de Michel Butor que Paola Viganò place en exergue de La Città elementare (Milan, Skira, 1999).

\section{Il suffit de penser à l'émergence,} dans les analyses sociologiques, des sujets individuels et collectifs, de l'autonomie revendiquée de leurs mouvements face à la très rigide stratification par classes des périodes précédentes. On peut aussi songer à la déconstruction analogue de la hiérarchie traditionnelle des (grandes) entreprises leaders et des petites et moyennes entreprises « induites » qui se sont constituées comme un monde autonome souvent plus entreprenant que celui de la grande entreprise. On peut encore faire référence à la décomposition sur une base spatiale des politiques macro en politiques micro, à la prolifération des politiques pour des lieux ou des événements spécifiques. 
elle-même n'apparaissait plus comme une entité s'opposant par une forme compacte à un extérieur. En synthétisant à l'extrême, on pourrait même rattacher une grande partie de l'histoire récente du projet urbain et du projet territorial à ce processus. Un des "problèmes ", par exemple, que les schémas conceptuels traditionnels n'expliquent pas et qui ont provoqué durant les dernières décennies une forte demande de théorie nouvelle, est constitué par la reconnaissance antérieure datant des années 1960 et 1970, du caractère composite de l'espace urbain, du collage dans lequel sont juxtaposées des parties morphologiquement reconnaissables (Rossi, 1966; Aymonino, 1971); ces parties représentent diverses hypothèses concernant les modes de vie possibles, les comportements ou caractéristiques des différents acteurs de la transformation de l'espace urbain; une caractéristique dont on pouvait commencer à considérer l'histoire sur une longue période (Rowe, Koetter, 1981), comme on pouvait aussi reconnaître la longue histoire de la dispersion de I'urbanisation (Secchi, 2005) dans des territoires qui pouvaient difficilement être encore pensés, dans une optique de projet, comme campagne urbanisée (Samonà, 1971). Dans l'incertitude théorique des dernières décennies du XXe siècle, tout cela a donné lieu, comme dans d'autres disciplines, à une sorte de boulimie lexicale: ville région, ville territoire, ville diffuse, network city, ville émergente, generic city, Zwischenstadt, ville franchisée... Tous ces termes ne sont certainement pas équivalents, mais ce sont ceux avec lesquels on a cherché à dire la nouvelle situation de la ville et du territoire européens ${ }^{5}$.

De nombreux travaux d'historiens et de géographes ont montré par ailleurs que, dans chaque subdivision du territoire comme dans la propre histoire de ce territoire, on devait reconnaître différents parcours qui se croisent et se superposent: histoire des communautés locales, ethniques et linguistiques; histoire de leurs organisations institutionnelles et de la restructuration cyclique des diverses économies nationales et régionales; histoire des sociétés et des mouvements sociaux, souvent conflictuels et caractérisés par des temporalités différentes; histoires de légalités et d'illégalités; histoires de richesse et de pauvreté qui rendent compte des différentes échelles identitaires.

Ces travaux ont également mis en relief les changements continuels, les croisements permanents et les fréquentes superpositions des échelles de pouvoir. Le pouvoir a toujours eu de substantielles difficultés à trouver une forme d'organisation stable dans le temps et dans l'espace, à s'organiser de façon cohérente face à la réalité des transformations territoriales de la société qu'il prétend contrôler, voire diriger et promouvoir. Depuis longtemps, les recherches urbaines et les projets d'urbanisme ont mis en évidence la contradiction profonde, à la fois irréductible et pérenne, entre les formes d'un pouvoir aspirant à la stabilité (sans laquelle aucun projet d'urbanisme à long terme ne peut exister) et les formes de la connaissance qui sont mobiles par nature (refusant systématiquement toute réduction et toute codification stables).

Ces mêmes recherches ont signifié l'importance d'un retour à l'expérience du quotidien, à la dimension corporelle de l'espace urbain et aux pratiques individuelles et collectives qui s'y déroulent. C'était une période où la géographie des espaces publics et privés connaissait des transformations rapides de plus en plus instables,
5. Voir Aldo Rossi, L'architettura della città, Padoue, Marsilio Editori, 1966; trad. fr., L'architecture de la ville, Paris, L'Équerre, 1981. Carlo Aymonino, II significato delle città, Bari, Laterza, 1971. Colin Rowe, Fred Koetter, Collage City, Cambridge (Mass.), MIT Press, 1978; trad. fr., Collage City, Paris, Éditions du Centre Georges-Pompidou, 1993; C. Rowe, "The Present Urban Predicament ", The Cornell Journal of Architecture, $\mathrm{n}^{\circ} 1$, 1981. Bernardo Secchi, La città del ventesimo secolo, Rome-Bari, Editori Laterza, 2005; trad. fr. La ville du Xx siècle, Paris, Éditions Recherches, 2009; Prima lezione di urbanistica, Bari, Laterza, 2000; trad. fr. Première leçon d'urbanisme, Marseille, Parenthèses, coll. «Eupalinos », 2006. Giuseppe Samonà, L'urbanistica e l'avvenire delle città negli stati europei, Bari, Laterza, 1971 [1959].

6. Hannah Arendt, The Human Condition, Chicago/Londres, University of Chicago Press,
1958; Condition de l'homme moderne, Paris, Calmann Lévy, coll. "Liberté de l'esprit », 1961. Jürgen Habermas, « Zur Logik der Sozialwissenschaften ", Philosophische Rundschau, supplément n 5, Tübingen, 1967 . rééd., Francfort, Suhrkamp, 1970; trad. fr., Logique des sciences sociales et autres essais, Paris, PUF, 1987. Richard Sennet, The Fall of Public Man, New York, Norton, 1976. Robert Ezra Park, Masse und Publikum. Eine methodologische und soziologische Untersuchung, Berne, Lack \& Grunau, 1904 ; 
occasionnant de nouveaux processus d'appropriation de I'espace individuel ou collectif. De fait, le concept de bien public, si important dans la construction du welfare state, s'est trouvé remis en cause. On peut d'ailleurs en dire autant de la notion même de public, tout aussi importante dans la dernière partie de la modernité, qu'il s'agisse de la version d'Hannah Arendt (1958), de celles de Jürgen Habermas (1967) et de Richard Sennet (1976), ou encore des versions bien plus anciennes de Robert Ezra Park (1904) ou Gabriel Tarde (1901).

Cette tendance a été renforcée par les nouvelles préoccupations environnementales et le rôle des espaces non bâtis dans la construction de réseaux écologiques efficaces, dans la gestion des réseaux de l'eau et de l'énergie, dans la restructuration des activités agricoles et dans les pratiques liées aux sports et au loisir. Grâce à ces projets et recherches focalisés sur la priorité environnementale, les transformations de la ville et du territoire ont mis en lumière des questions importantes relatives à la porosité, à la perméabilité physique et fonctionnelle: activités, individus et groupes sociaux, eau, végétaux ou animaux semblent traverser le bâti comme on passe à travers un filtre, dans un mouvement général et permanent. Ils traversent l'espace urbanisé comme une éponge, dans un mouvement de percolation qui suscite souvent des ambiguïtés, des contradictions et des conflits ouverts. Or, ce qui caractérise le phénomène de percolation, c'est que tout ce qui passe à travers comme tout ce qui est traversé change de statut dans un mouvement continuel, plus ou moins rapide, de transformation. Ceci a conduit à porter un regard différent sur les dispositifs de passage entre les échelles macro et micro: il ne s'agit pas tant de passer de visions lointaines à des visions rapprochées que d'explorer les changements de statut et de rôle d'un même matériau, physique ou social, dans des contextes et des systèmes de relations différents.

\section{Ce que nous avons appris de l'approche bottom up}

À partir de ces expériences, nous, architectes et urbanistes, sociologues et économistes, géographes et historiens, avons donc appris à re-conceptualiser un certain nombre de thématiques fondamentales. Par exemple, nous avons compris qu'il est nécessaire de:

\section{- re-conceptualiser l'habiter.}

II faut reconsidérer I'habiter comme pratique et comme représentation dans l'espace de la ville diffuse ainsi que dans l'espace de la ville consolidée avec ses périphéries. Les territoires de la dispersion, par exemple, auxquels j'attribue une grande importance, occupent désormais une part consistante du continent européen, avec des caractéristiques variables selon les différentes régions. La dispersion de l'urbanisation dans la " ville diffuse ${ }^{7}$ " et I'usage extensif de vastes territoires (voire d'une seule ville) ont connoté la dernière partie du $\mathrm{XX}^{\mathrm{e}}$ siècle et cela représente, de mon point de vue, un phénomène de même portée que la réalisation des grandes périphéries métropolitaines, de même envergure que la construction des grandes aires industrielles au $X X^{e}$ siècle, de même importance que la croissance des villes industrielles au $\mathrm{XIX}$ siècle.

La dispersion ne concerne pas seulement la modification des aspects physiques de la ville européenne: elle trad. fr., La foule et le public, Lyon, Parangon/Vs, 2007. Gabriel Tarde, L'opinion et la foule, Paris, PUF, 1901

7. J'emploie ce terme, que j'avais proposé initialement avec Francesco Indovina (F. Indovina, La città diffusa, Venise, Dipartimento di analisi economica e sociale del territorio, Istituto Universitario di Architecttura, 1990), parce qu'il me semble le mieux mettre en lumière les caractéristiques des situations auxquelles il est fait référence: de vastes territoires connotés par une urbanisation dispersée qui n'est pas la conséquence d'un spill over de la ville centrale et qui est utilisée par la population qui I'habite (une population qui n'a jamais habité dans la ville centrale ou dans l'une des villes mineures extérieures) comme une unique grande ville dans laquelle les distances entre les matériaux qui la composent sont devenues énormes, si on les confronte à celles de la ville compacte; une ville de basse densité grâce à ces immenses dilatations de distances plus encore qu'en raison des formes spécifiques des bâtiments qui la constituent. Dans l'usage élargi du territoire qui connote les pratiques individuelles et collectives, les concepts de proximité et de distance acquièrent des significations complètement différentes des sens traditionnels: souvent, le lieu de travail et l'habitation des amis ou des parents sont proches, tandis que les équipements ou les lieux urbains, l'hôpital et l'université, le centre commercial ou 
concerne aussi la modification des relations entre les différents groupes sociaux, le rôle des différents acteurs, les comportements individuels ou collectifs, les structures institutionnelles. Ne pas prendre en compte cette dispersion et s'y opposer par un retour à la ville compacte de l'Ancien Régime ou de la période moderne (ne pas saisir les opportunités qu'elle suggère et ne pas chercher à en résoudre les problèmes) est illusoire, parfois pathétique et souvent réactionnaire.

La difficulté à accepter cette nouvelle situation, devenue rapidement un objet de condamnation, devrait aussi être expliquée, au moins comme un phénomène digne d'étude. Le socle idéologique de ce refus, souvent caché derrière d'évidentes considérations esthétiques, me semble profondément enraciné dans la culture des groupes sociaux dont le capital culturel et social s'appauvrit progressivement ${ }^{8}$. Il est en effet tout à fait évident que la ville dispersée n'est pas « belle »; que les exemples de bonne architecture urbaine y sont rares; que très souvent, elle n'arrive même pas à exprimer un caractère vernaculaire d'un quelconque intérêt; que, confrontée aux parties de villes du passé, souvent aussi critiquées par leurs contemporains, elle présente une lisibilité bien moindre de l'espace urbain, une moindre articulation, une absence presque totale d'éléments qui confèrent à ses différentes parties un sens partagé. Mais peut-être faut-il aussi reconnaître qu'aucune ville ne naît belle, avec des monuments et des espaces que nous ne pouvons admirer qu'après plusieurs siècles. Si l'on excepte certaines villes de fondation, toutes les villes naissent comme un amas de cabanes, de baraques, de médiocres structures construites et ensuite, au fil du

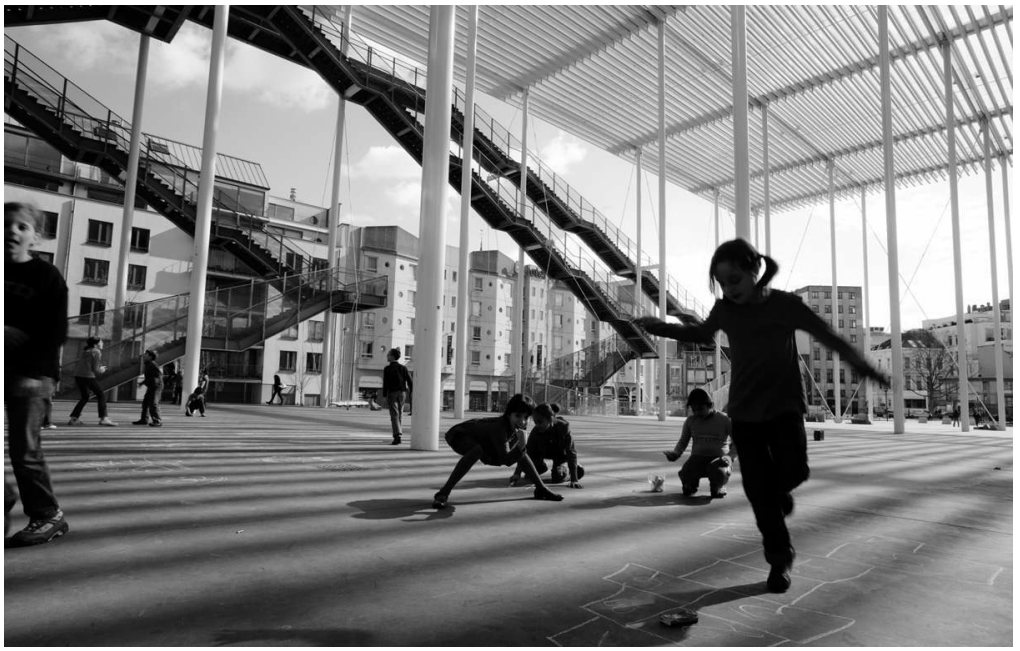

la discothèque sont dispersés et éloignés, entraînant une évidente modification des pratiques spatiales traditionnelles. Barthes aurait parlé d' "idiorythmes ", ceux qu'observent les études de la mobilité et de ses rythmes journaliers ou hebdomadaires. Voir Roland Barthes, Comment vivre ensemble. Cours et séminaires au Collège de France, (1976-1977), texte établi, annoté et présenté par Claude Coste, Paris, coédition Seuil / Imec, 2002.
8. Pierre Bourdieu, La distinction. Critique sociale du jugement, Paris, Les Éditions de Minuit, 1979

9. Elles sont toutes articulées et connotées de façon différente. En Vénétie, par exemple, il me semble possible de reconnaître, comme Banham, une écologie des grandes infrastructures, une écologie de la ville compacte et de ses banlieues modernes, une écologie des zones de dispersion. II ne s'agit pas de «zones »
Anvers.

La place des théâtres est un espace utilisé de différentes manière: par exemple, comme espace de jeu par les enfants. Il s'agit d'un projet construit à partir soit d'une approche bottom up, soit, pour ce qui concerne son rôle dans la ville, d'une approche top down. clairement distinctes; les superpositions et les croisements sont nombreux; elles peuvent être observées soit dans leur constitution physique de ville diffuse, soit dans les pratiques sociales et, surtout dans ce que j'ai appelé l'" usage élargi du territoire ", c'est-à-dire l'usage de ce vaste territoire (mais comme également dans les Flandres ou d'autres régions européennes) comme d'une ville unique. Voir Reyner Banham, Los Angeles: the Architecture of four Ecologies, [New York, Harper et Row, 
temps, elles réussissent à identifier des lieux remarquables, à exprimer une signification par des architectures spécifiques, à devenir des complexes dotés de sens et de lisibilité. Si l'on observe le sens de nombreux projets urbains de ces dernières décennies, au-delà des intentions de leurs auteurs, des objectifs et de la conscience de ceux qui les ont promus, on peut reconnaître que les grandes périphéries de la ville moderne qui, jusqu'à récemment, étaient regardées avec tant de mépris, sont maintenant dans une phase de reconnaissance en tant que lieux significatifs où devient possible, parce qu'elle est partagée, une action de signification; cette phase, qui peut se dérouler sur plusieurs décennies, aboutit habituellement à un processus de refonctionnalisation, de densification physique, fonctionnelle et symbolique et de construction d'une nouvelle image.

Les territoires de la dispersion n'en sont pas encore là, mais c'est peut-être à nous de penser à ce que devraient être leurs caractères futurs, probablement assez différents de ceux de la ville du passé. Les territoires de la dispersion donnent peut-être lieu aujourd'hui à une nouvelle «forme de ville», probablement plus avancée que les précédentes au sens où elle les englobe toutes sans les contredire et en leur donnant de l'espace. En paraphrasant Reyner Banham (1971), on pourrait dire qu'ils donnent lieu à une ville de diverses écologies ${ }^{9}$, qui ajoute à la ville européenne quelque chose de profondément cohérent avec la société et l'économie contemporaines. Ce qu'ajoute la dispersion, c'est un extraordinaire élargissement des opportunités de choix des styles de vie et des modes d'habiter. De ce point de vue, il convient de citer des cas exemplaires comme les Flandres ou la
Vénétie ${ }^{10}$, la Bretagne ou le nord du Portugal, et bien $d$ 'autres également qui sont en cours de constitution dans de nombreuses régions européennes, y compris dans les pays de l'Est, quelles que soient leurs différences.

En même temps, la ville ancienne et la ville moderne sont progressivement devenues de plus en plus poreuses. L'abandon d'un grand nombre de zones productives constitue une cause importante mais pas unique de ce phénomène qui, par surprise, a engendré dans les tissus urbains depuis longtemps consolidés de grandes mutations morphologiques, fonctionnelles et symboliques.

Une attitude inspirée du pragmatisme de l'urban market a souvent dominé. Elle s'est nourrie d'une réalité issue de la situation économiquement grave de nombreuses régions urbaines et de la pression exercée par de puissants groupes d'intérêt. Elle a souvent empêché une réflexion plus approfondie, capable de saisir les opportunités de redéfinition sociale, fonctionnelle et symbolique qu'offre l'extraordinaire quantité de zones et infrastructures devenues tout à coup disponibles. On a seulement cherché à coloniser ces espaces par une série de projets qui ont recouru à des images, à des langages, à des contenus très dispersés, accentuant le caractère fragmentaire qui depuis toujours connote la ville ${ }^{11}$.

En même temps, dans la ville européenne comme ailleurs, les phénomènes de séparation, d'exclusion et de marginalisation se sont accentués. D'un côté, ils ont assumé comme référence au moins implicite les gated communities des deux continents américains et de l'Est asiatique. De l'autre côté, ils ont assumé la mise à l'épreuve
1971], Berkeley, University of California Press, 2001.

10. Naturellement, la ville diffuse a aussi fait l'objet de critiques plus sérieuses que celles qui sont fondées uniquement sur un jugement esthétique: les plus importantes ont porté sur la "consommation des sols" et sur les consommations d'énergie. On entend généralement par consommation des sols la quantité de sol urbanisé soustraite à d'autres usages, principalement à une destination agricole. Cet argument devient ambigu dans des régions où la production agricole est tout à fait marginale et ne résiste que grâce à d'importantes aides publiques. Par exemple, dans les zones de la ville diffuse de la Vénétie, le territoire agricole couvre $78 \%$ de la surface totale, mais il ne contribue que pour $2 \%$ à la formation du produit intérieur brut de cette région. II faudrait peut-être observer d'autres indicateurs: par exemple, pour un même nombre d'habitants, les quantités de sols imperméables de la ville compacte et les quantités de sols perméables de la ville diffuse.

11. Certaines recherches et plusieurs projets réalisés à une échelle micro ont toutefois montré que dans la porosité se produisent également des phénomènes intéressants, peut-être riches de conséquences sur le long terme; en particulier, de nouvelles formes diffuses de l'habiter considérées comme de nouvelles configurations 
de la flexibilité des stocks résidentiels construits pour des groupes sociaux aisés puis abandonnés à de nouveaux immigrés. Ce processus de mutation, qualifié de filtering down, se poursuit jusqu'au moment où le stock résidentiel est récupéré à travers un nouveau phénomène de "distinction » et de gentrification, comme une vague dont chaque passage imprègne les tissus urbains, modifiant le statut de ses structures physiques et sociales. Les exemples que l'on peut évoquer sont assez nombreux: le quartier de la Goutte d'or, à Paris, est l'un d'eux.

Dans une période de croissance des inégalités sociales et de rapide dissolution du welfare state, ceci a donné lieu - et donne lieu de plus en plus souvent - à une reconfiguration de la structure sociale de la ville européenne. Des géographies sociales nouvelles et inattendues comme des écologies nouvelles et inattendues traversent la ville ancienne, la ville moderne et la ville diffuse sans respecter les modèles que l'on pensait consolidés par la tradition. Ces phénomènes agissent comme des éléments de perturbation et de fragmentation des identités sociospatiales (et par conséquent, des marchés foncier et immobilier); ils ont recours à des dispositifs spatiaux qui traversent manifestement l'ensemble du monde globalisé contemporain.

L'espace de l'habitation est constitué par un ensemble de matériaux, internes et externes au logement, et présents dans tout l'espace urbain. Ils peuvent être identifiés, isolés et assemblés de plusieurs façons, selon différentes séquences et densités. L'histoire de la ville européenne en a codifié certains, qui sont aujourd'hui encore très visibles: la maison bourgeoise, avec ses rapports à l'espace de la rue et à l'espace de la cour, est
I'un des nombreux exemples qui manipulent ces matériaux. Mais ce qui est en train de se produire dans les territoires contemporains (dans la ville diffuse comme dans la ville consolidée avec ses banlieues), c'est que chaque sujet social compose les mêmes matériaux à sa façon et chaque sujet social utilise aussi (souvent en les modifiant) des compositions élaborées par d'autres, qui I'ont précédé ou qui ont voulu interpréter son style de vie. Traditions et préjugés, ambitions et frustrations, limites et ressources disponibles se conjuguent comme d'habitude dans les pratiques et les imaginaires collectifs. Tout cela joue bien sûr un rôle déterminant, mais empêche souvent une meilleure adhésion entre les habitants et les composants de l'espace urbain. Seules des recherches micro (Lanzani, $2007^{12}$ ) peuvent analyser cette réalité, sans toutefois résoudre les sérieux problèmes posés à chaque fois que l'on affronte la construction d'une politique de la mixité capable de s'affirmer sur un temps long.

\section{- reconceptualiser les rapports entre ville et productivité.}

Il est important de préciser que la dispersion de l'urbanisation sur de vastes territoires n'est pas seulement issue du processus de décentralisation productive et d'externalisation accélérée de certaines phases de production des petites et moyennes entreprises. II est tout aussi nécessaire de dire que la porosité de la ville contemporaine n'est pas seulement issue de l'abandon des lieux congestionnés par beaucoup d'entreprises à la recherche de plus grands espaces. II convient enfin de souligner que l'urbanisme diffus des dernières décennies n'est pas de l'espace interne à l'habitation, de ses nouvelles relations avec l'espace extérieur de proximité, avec les principaux équipements urbains et/ou avec l'espace urbain tout entier. Voir P. Viganò, On porosity, communication à la conférence PermaCity, Delft, 27-28 novembre 2007 (TUDelft, 2007). Ces formes, pour le moment minoritaires, parfois élitaires, mais toujours plus diffuses, constituent la véritable critique des modes d'habiter d'une société de masse qui ont connoté les périphéries urbaines de l'après-guerre; elles suggèrent des recherches et des approfondissements de portée analogue à ceux qui ont été réalisés en Europe dans les années 1910 et 1920.

12. Voir Arturo Lanzani, Elena Granata (dir.), Esperienze e paesaggi dell'abitare. Itinerari nella regione urbana milanese, Milan, AimSegesta, Multiplicity lab, 2006 ; Milano, cronache dell'abitare, Milan, Bruno Mondadori, 2007. 


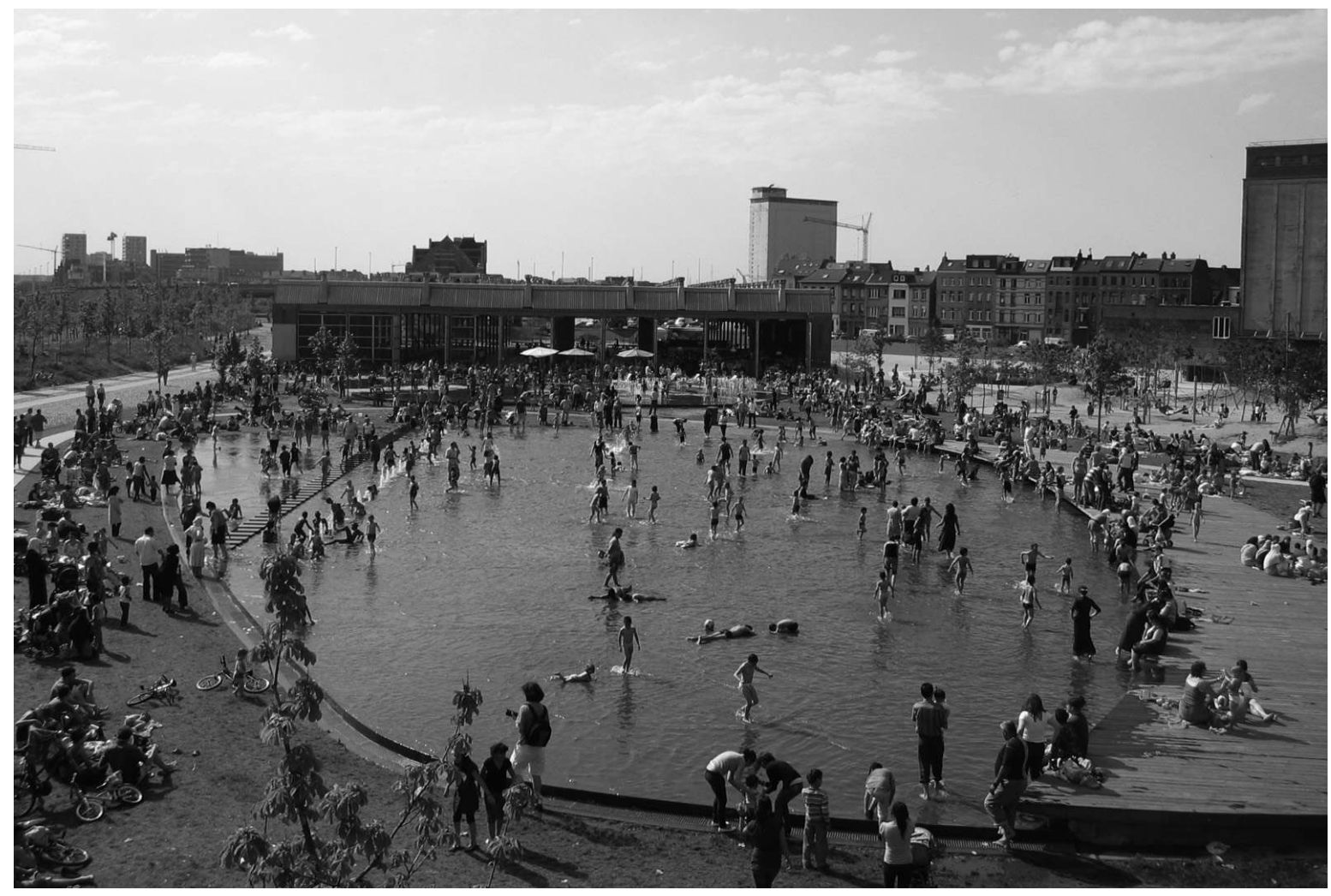

En haut: Anvers.

Spoornoord un jour

d'été : les résultats

d'une approche

bottom up.

En bas: Anvers.

Maquette du parc

Spoornoord.

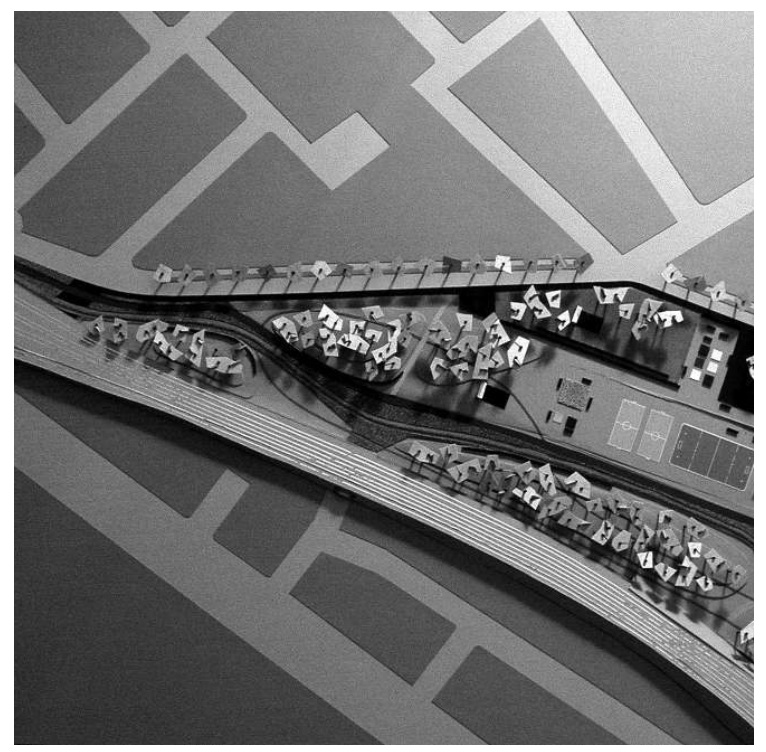


seulement le fruit des restructurations de l'économie industrielle occidentale avec ses délocalisations massives dans d'autres parties du monde.

En fait, plusieurs autres raisons sont à l'origine de la dispersion de l'urbanisation dans les régions européennes: I'apparition dans de nombreux secteurs industriels d'entreprises diffuses, souvent autonomes et enracinées dans des "savoirs contextuels » spécifiques; la recherche d'un habitat plus proche du lieu de travail et de la «nature»; la discontinuité des trends démographiques; le vieillissement progressif de nombreuses régions européennes; l'abandon des zones centrales de la ville aux nouvelles populations immigrées. Chacune de ces tendances s'est ensuite développée de manière différente pour les groupes de population et groupes d'entreprises concernés. Dans de nombreuses régions, la dispersion productive des dernières décennies du $X x^{e}$ siècle a établi avec la ville et le territoire des rapports insolites et pas toujours vertueux. Elle a rendu obsolètes les formes originelles de la ville industrielle (avec son support infrastructurel) et les formes successives de « ville-usine » (dont les traces sont encore souvent évidentes).

La dispersion résidentielle et la dispersion productive

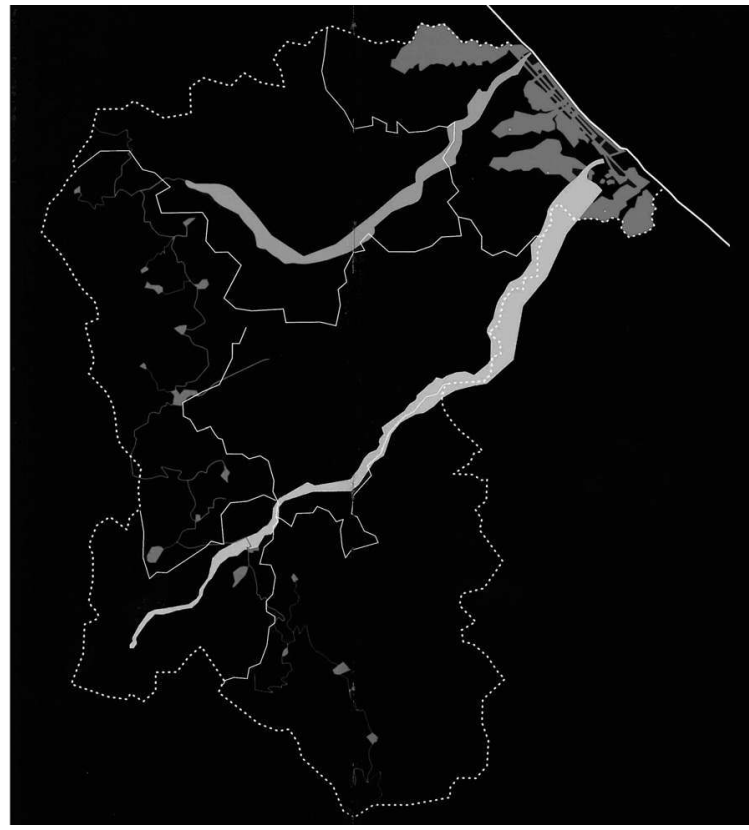

Les espaces stratégiques

dans le plan territorial de la province de Pescara (Italie). ont surtout été, au moins au début, des phénomènes de capital saving, c'est-à-dire des réflexes d'utilisation du capital fixe existant (routes, canaux, aqueducs, réseaux d'égouts, réseaux électriques et téléphoniques) qu'il a fallu étendre et compléter. Mais l'infrastructuration du territoire a presque toujours été conçue de façon réductrice comme une simple augmentation de l'offre d'infrastructures pour la mobilité. Elle n'a pas été conçue pour assumer quantitativement et conceptuellement le

13. La dispersion des zones productives, apparemment arbitraire mais en réalité déterminée en grande partie par de forts localismes qui ont marqué les dernières décennies et par l'épaisseur de leurs divers aspects (historique, identitaire...). Cette dispersion contredit de façon évidente la critique du zoning des années passées et permet de comprendre comment le zoning, c'est-à-dire l'individualisation dans la ville et le territoire de zones fonctionnellement ou socialement caractérisées, a de fait une histoire plus longue; cela remonte au moins au XVIII siècle (B. Secchi, 2005), c'est-à-dire bien avant sa codification par des pratiques urbanistiques et administratives. Mais le caractère même de ces zones, désertes le soir et les jours fériés, dispersées et donc difficilement desservies par les réseaux des grandes infrastructures, soulève, dans des périodes dominées par les rhétoriques de la mobilité et de la peur, des réactions opposées à une plus grande mixité fonctionnelle et d'une localisation plus logique par rapport aux réseaux d'infrastructures: des questions qui demandent précisément des réflexions à plusieurs niveaux. 
phénomène de la dispersion. Même au début, alors que naissait un processus de croissance et de sélection cumulative des différentes entreprises sous l'effet NIMBY, alors que l'on cherchait à encourager une relative concentration des activités productives dans des platesformes industrielles indifférentes au contexte, la question de la dispersion n'a pas pu être maîtrisée. Dans des territoires habités par la douceur de l'histoire se sont alors installés, de manière apparemment aléatoire, des corps étrangers, des " cailloux », des objets solides et durs préoccupés par leur propre imperméabilité physique, fonctionnelle et symbolique ${ }^{13}$.

Tous les secteurs de production font désormais l'objet de profondes restructurations qui épousent la globalisation croissante du Marché. Les raisons qui ont donné lieu à une nouvelle géographie de ces secteurs de production à partir des années 1970 importent peu car, désormais, les nouvelles entreprises ont donné priorité à de nouvelles stratégies de reconnaissance. II devient ainsi toujours plus difficile de distinguer les productions de marchandises et les productions de services, les productions propres et les productions polluantes, les productions destinées aux marchés locaux et les productions destinées aux marchés globaux. Il devient aussi toujours plus difficile de concevoir des stratégies d'occupation de l'espace en suivant les théories traditionnelles de la localisation car, compétitivité oblige, les groupes industriels et les entreprises conjuguent un calcul économique élargi à l'échelle des enjeux mondialisés avec des arguments liés au contexte local des politiques publiques, des réalités sociales, des perspectives financières, des enjeux immobiliers et des contraintes environnementales. Tous ces arguments participent évidemment à la construction savante de l'image de chaque groupe industriel et de chaque entreprise.

\section{- reconceptualiser la mobilité.}

Les réponses apportées à une demande croissante de mobilité ont été banales, grossières, souvent illusoires et inadaptées aux changements de la société. Mais elles ont produit une transformation conceptuelle radicale des relations entre les infrastructures de la mobilité et le territoire.

Reprenant des idées qui avaient été celles de Diderot, de Lamarck, de Quesnay et de Saint-Simon, on a de plus en plus souvent conceptualisé la société et le territoire comme des « réseaux de réseaux » dont l'évolution serait éminemment déterminée par la nature et l'intensité des flux qui les parcourent ainsi que par leur « capacité ${ }^{14}$ à permettre la circulation.

Cette tendance doit beaucoup à l'étude de la dynamique des fluides et aux convergences de l'hydraulique, de la physiologie et de l'économie politique. Sa banalisation a considéré de façon très réductrice qu'il fallait répondre à la demande de mobilité par des infrastructures de dimensions toujours plus importantes, conçues comme de grands "tubes " susceptibles de canaliser des flux croissants de personnes, de biens et d'informations. Négligeant la notion de « capacité » conçue comme aptitude, on a donc cherché à passer de réseaux très connexes (spongieux comme ceux qui permettent la circulation du sang dans le cerveau) à des réseaux « en arbre », fortement hiérarchisés, dont la capacité augmente avec le niveau hiérarchique. Imperméables à l'extérieur (à

14. Dans la terminologie scientifique de la fin du XVIII ${ }^{e}$ siècle et du début du XIX", de Diderot, Lamarck, Quesnay et SaintSimon, le terme de capacité a le double sens d'aptitude et de possibilité de contenir (contenance) par ce qu'ils indiquaient comme des «tubes » et qui pouvaient être des artères, des veines, des canaux, des systèmes d'échange et de transport de marchandises: Pierre Musso, La religion du monde industriel. Analyse de la pensée de SaintSimon, Paris, Éditions de l'Aube, 2006. 


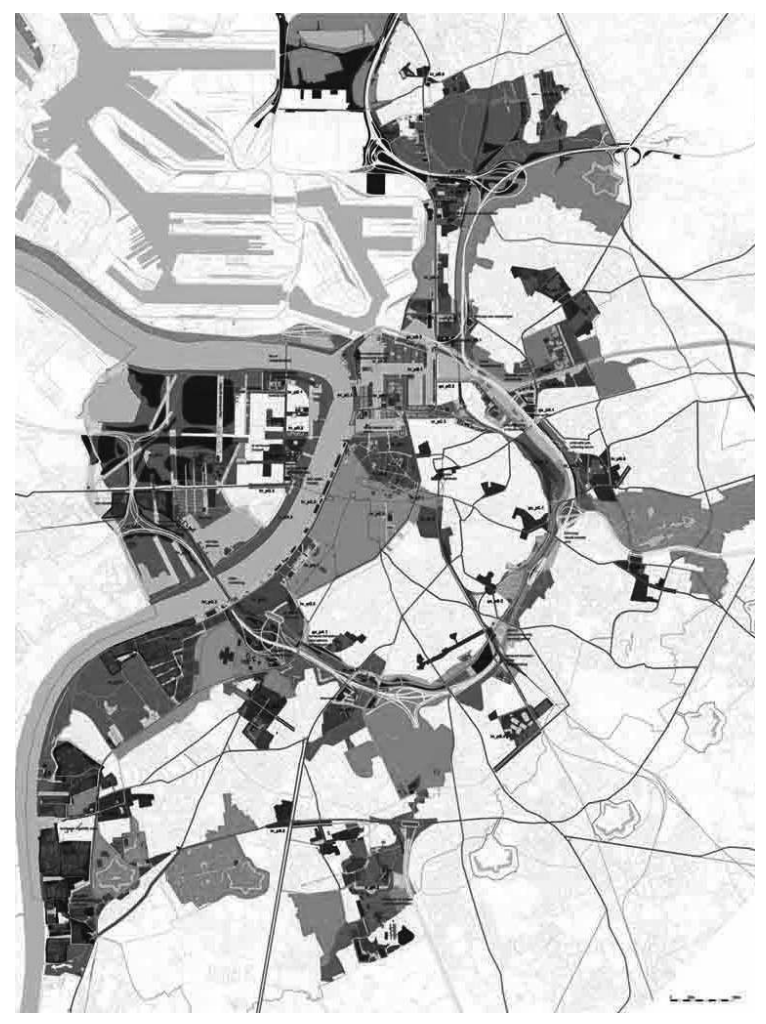

Les espaces stratégiques et les projets stratégiques (en foncé): la place des théâtres et le parc Spoornoord ont été considérés comme des projets stratégiques. La carte stratégique est ce qui lie, dans les plans de Studio Secchi-Viganò, les deux approches: bottom up et top down. l'exception de quelques échangeurs), ils se sont opposés aux rapports d'osmose que les "réseaux spongieux" établissent avec le contexte. Un phénomène analogue s'était d'ailleurs déjà produit dans le domaine hydraulique: les "réseaux spongieux » sur lesquels (et grâce auxquels) s'était constituée la ville diffuse ont alors été considérés de façon erronée comme le résidu des temps passés. Les grandes infrastructures de transport, quant à elles, sont devenues des barrières qui connectent des points distants l'un de l'autre mais qui séparent des aires urbaines et des territoires souvent liés par des réseaux relationnels denses. En tant que telles, et pas seulement dans des cas extrêmes, elles sont utilisées (Petti, 2007 ${ }^{15}$ ) par une politique d'exclusion et de séparation qui redessine la géographie économique sociale de la ville et du territoire.

\section{- reconceptualiser l'infrastructure.}

Les expériences de ces dernières années ont montré la nécessité d'un concept d'infrastructure bien plus large que celui qui a généralement présidé aux politiques urbaines et territoriales. Elles ont démontré en particulier que le concept d'infrastructure ne peut plus se limiter aux seules infrastructures de la mobilité - qui, en l'occurrence, devraient intégrer les personnes, les choses, l'énergie, I'information, etc., c'est-à-dire l'espace entier des flux (Castells, 1989, 2002, 2004 ${ }^{16}$ ). Mais si par infrastructure on entend ce qui permet le déroulement continu du processus de reproduction sociale, alors tout le territoire (avec l'épaisseur de tous ses aspects matériels, symboliques, fonctionnels et esthétiques) doit être considéré comme une infrastructure fondamentale et irréductible.
15. Alessandro Petti, Arcipelaghi e enclave. L'architettura dell'ordinamento spaziale contemporaneo, Milan, Bruno Mondadori, 2007.

16. Manuel Castells, The Informational City, Oxford, Cambridge (Mass.), Blackwell, 1989 ; trad. fr. L'ère de l'information (vol. I, La société en réseaux; vol. II, Le pouvoir de l'identité; vol. III, Fin de millénaire), Paris, Fayard, 1998/1999. The Information
Society And The Welfare State: The Finnish Model, Oxford, Oxford University Press, 2002. La Galaxie Internet, Paris, Fayard, 2002. The Network Society: A Cross-Cultural Perspective, Northampton (Mass.), Edward Edgar Publishers, 2004. Communication Power, Oxford, Oxford University Press, août 2009. 


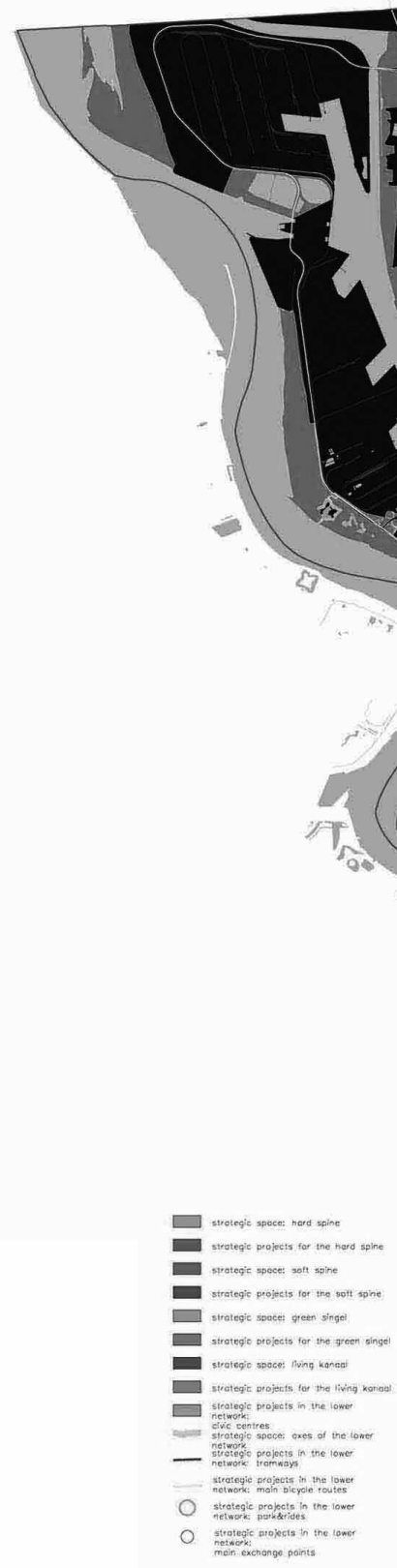

Plan structurel d'Anvers: espaces stratégiques (espaces dans lesquels les interventions majeures sont concentrées). La hard spine, la soft spine (cinq parcs liés entre eux et au fleuve); le singel (transformation de la rocade en un parc linéaire); le lower network (les réseaux de rues commerçantes et piétonnes) qui connecte les différents centres des quartiers. 
188 Les Cahiers de la recherche architecturale et urbaine - n²4/25 La critique en temps et lieux

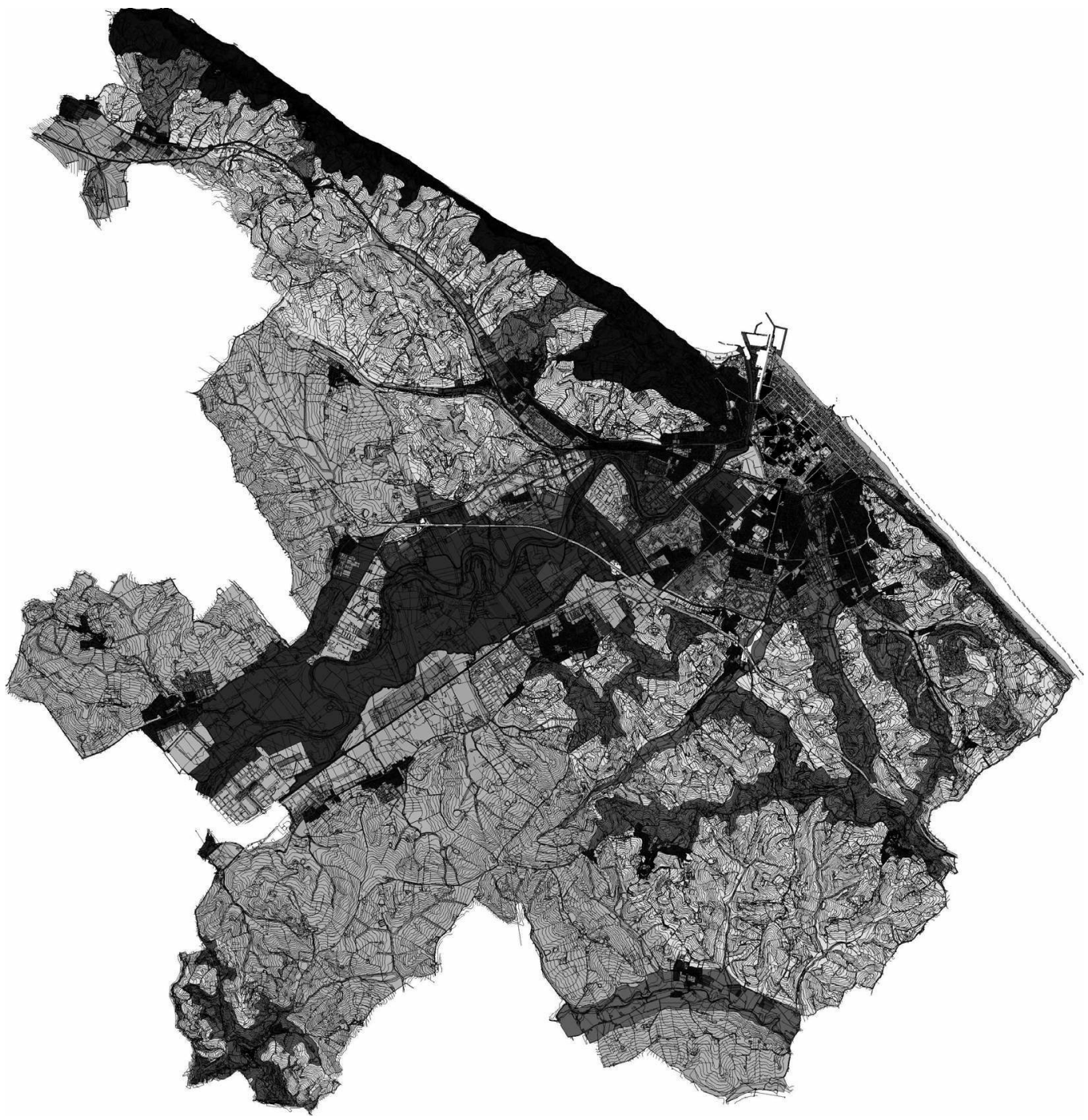

Structure spatiale

proposée pour le plan de Pesaro (Italie) : une approche top down. 


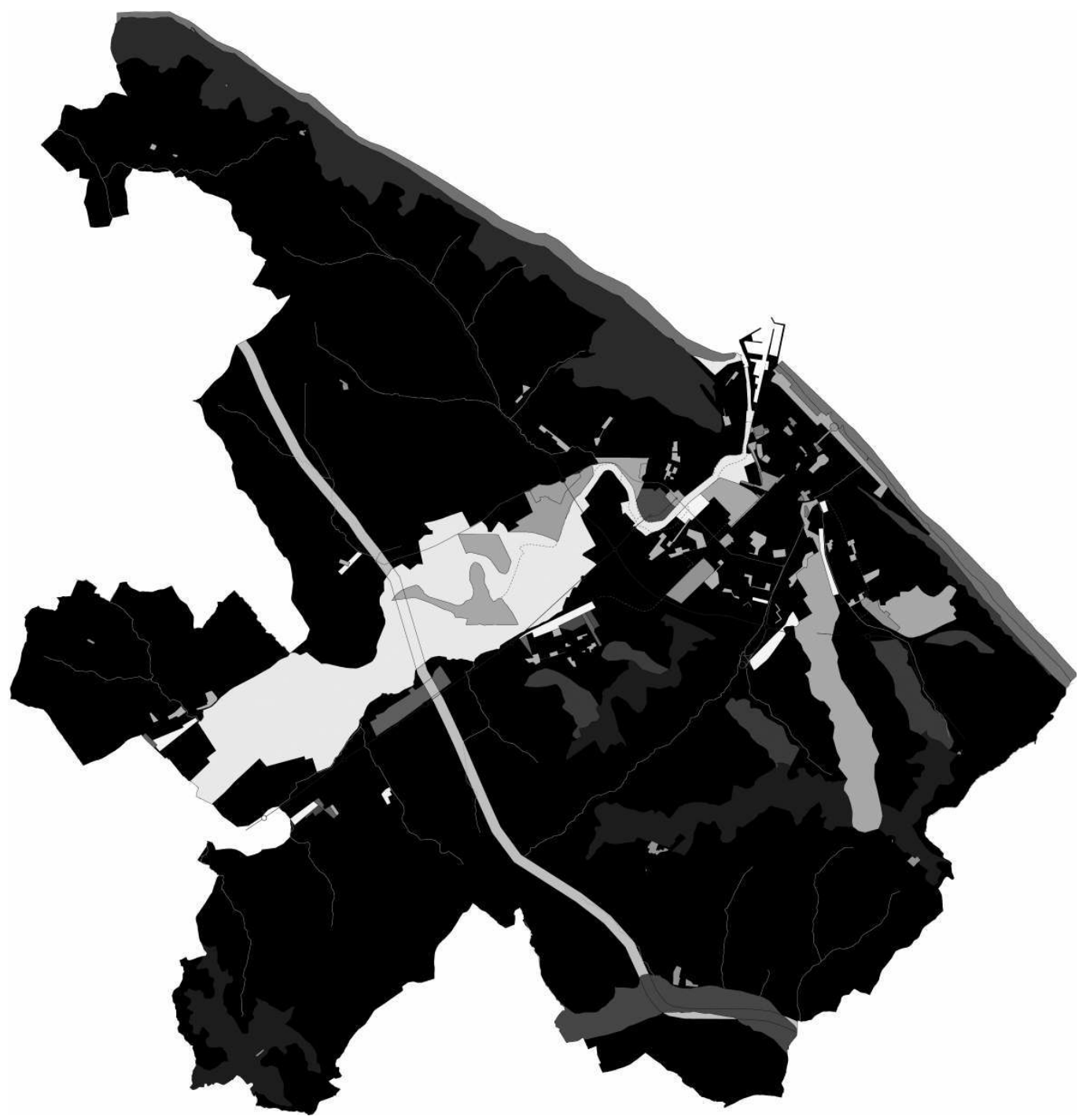

Carte stratégique du plan de Pesaro (les interventions jugées plus importantes, du point de vue de la réalisation de la nouvelle structure spatiale du territoire et prioritaires) : encore une fois, les deux approches se croisent. 
Les différents systèmes infrastructurels (voies ferrées, canaux, lignes électriques, réseaux de transports urbains, etc.) ont été au contraire traditionnellement pensés, conçus et réalisés indépendamment les uns des autres et dans une réciproque indifférence, ou presque. En ce qui concerne les ressources humaines mobilisées pour cela, on a assisté entre autres à la formation de corps techniques et administratifs, et de cultures techniques communiquant peu entre eux, et rarement de façon claire et responsable ${ }^{17}$.

Or les expériences réalisées ces dernières années ont montré que l'efficacité de chaque système profite de la conception d'une infrastructure unitaire et générale de l'économie et de la société. Ainsi, les infrastructures de proximité peuvent interagir avec les infrastructures de plus grande portée, les infrastructures écologiques avec les infrastructures énergétiques. Par exemple, la perméabilité ou l'imperméabilité des sols peut interagir avec les réseaux hydrauliques...

D'ailleurs, l'énergie, l'eau et la végétation jouent aujourd'hui et joueront demain un rôle tout aussi important (voire plus) que les routes et les voies ferrées, surtout dans un futur marqué par la rareté des ressources fossiles. Elles auront vraisemblablement des conséquences aussi grandes sur l'urbanisation que les infrastructures de transport traditionnelles.

\section{Top-down: trois scénarios et leurs conséquences}

Un auteur bien inspiré a pu affirmer que « faire des prévisions est toujours très difficile et dangereux, surtout quand elles concernent le futur ». II est toutefois possible de dire que trois scénarios menacent notre planète, avec des caractéristiques qui varient pour chacun selon les différentes parties du monde. Le premier est le scénario du global change, et en particulier du warming climate; le deuxième est celui d'une urbanisation croissante conduisant pour les prochaines décennies à la concentration de la majeure partie de la population mondiale dans de vastes zones urbaines; le troisième est celui de l'inégalité croissante entre les riches et les pauvres.

Ces trois scénarios doivent être liés, chacun pouvant être interprété comme la conséquence de l'autre. L'urbanisation croissante sera ainsi la cause et la conséquence des changements climatiques. L'inégalité croissante entre les riches et les pauvres sera aussi la cause et la conséquence du développement de l'urbanisation et des changements climatiques. Le caractère de chaque scénario est par ailleurs profondément ambigu car la constellation d'intérêts et les lignes d'action impliquées sont d'une interprétation difficile. Par exemple, I'urbanisation progressive et rapide de la planète qui soulevait à la fin du XIXe siècle une véritable angoisse diffuse est vue aujourd'hui comme un vecteur important de modernisation des couches sociales restées en marge de la modernité. Mais elle peut également être interprétée comme la voie royale de la formation de vastes territoires de consommation unifiés, apanage des grands groupes industriels qui agissent à l'échelle globale. Un autre exemple: le welfare state que nous avons surtout connu en Europe au Xx $x^{e}$ siècle se révèle incapable d'affronter et de résoudre les problèmes d'une société très différente de celle pour laquelle il avait été initialement conçu. Mais la destruction acritique du welfare state au nom d'une
17. Un des modes de communication, acritique et partiellement inconscient, des corps de techniciens et d'administrateurs souvent indifférents les uns aux autres, est constitué par le recours fréquent à des métaphores empruntées à d'autres champs disciplinaires et techniques; par exemple, I'utilisation de métaphores et de termes empruntés à l'hydraulique ou à la physiologie par les experts des transports et des infrastructures de mobilité. Saint-Simon a peut-être été l'un des premiers à souligner la productivité conceptuelle de ces aspects. 
CONSULTATION INTERNATIONALE DE RECHERCHE ET DÉVELOPPEMENT SUR « LE GRAND PARI DE L'AGGLOMÉRATION PARISIENNE ".

Arpenter le territoire, faire l'expérience des banlieues, parler avec les gens qui y habitent, les interroger, essayer de comprendre leurs imaginaires: cela a été notre stratégie de connaissance du Grand Paris, ce qui nous a amenés à reconnaître dans la grande métropole trois problèmes principaux: problèmes environnementaux, de gestion des eaux, de la biodiversité et de l'énergie; problèmes de la mobilité, comment donner aux habitants du Grand Paris une accessibilité généralisée ; problèmes liés aux inégalité sociales, comment désenclaver toutes les zones des banlieues. Reconnaître des problèmes n'est que le premier pas pour essayer de les résoudre, plusieurs exercices montrent que cela est possible. Par exemple le grand projet de la « traversée verte " dit comment restructurer l'espace urbain du Grand Paris, en sortant de l'idée qu'il s'agit nécessairement d'un espace radio-concentrique.

B. SECCHI

(C) Illustrations Secchi-Viganò
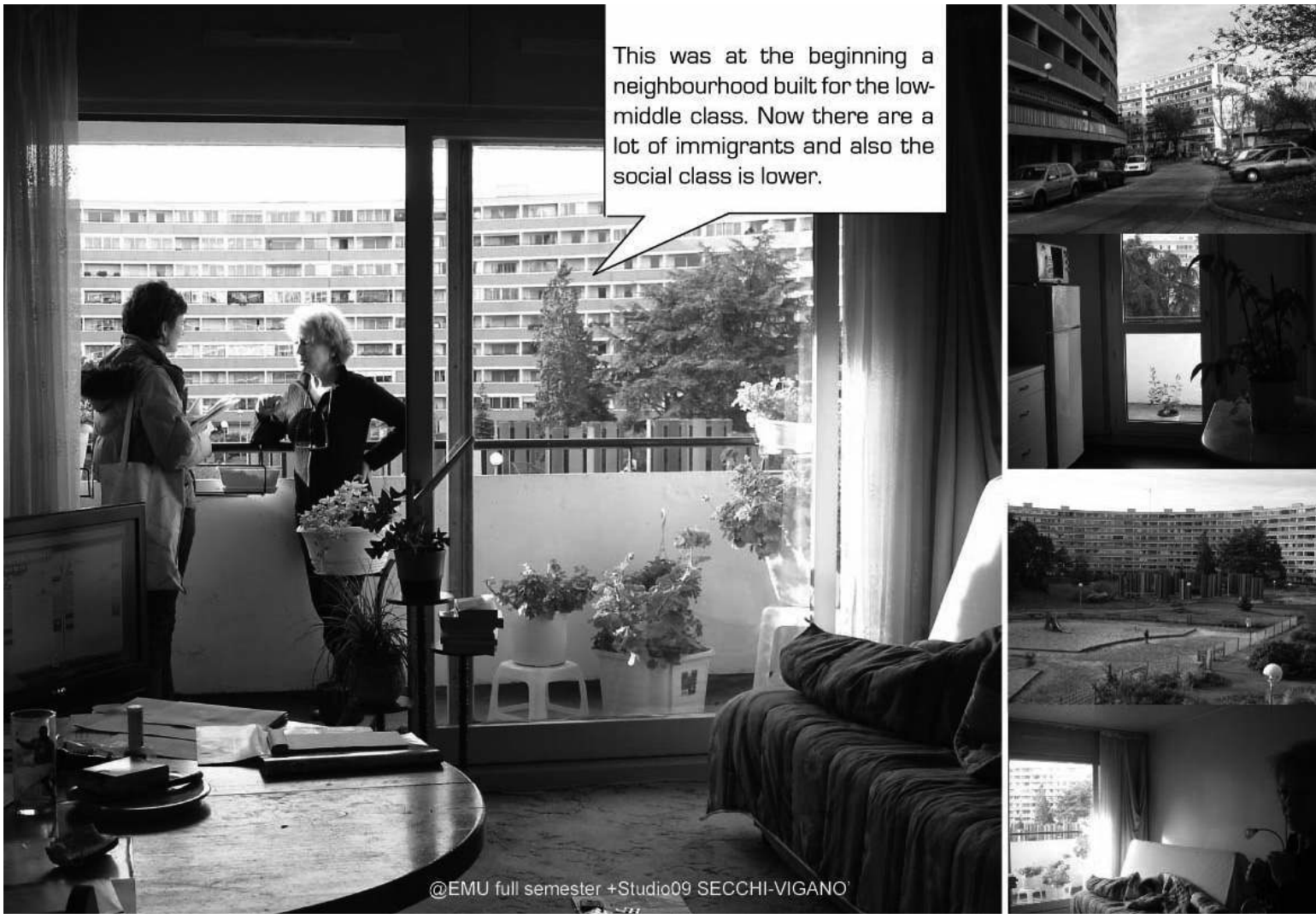
mythologie du Marché récompense toujours les uns et pénalise toujours les autres. On peut dire enfin que les changements climatiques n'agissent pas de façon homogène sur les perspectives de développement des différentes parties du monde et sanctionnent très probablement les régions les plus pauvres.

Éviter les pires conséquences de ces trois scénarios implique évidemment la mise en œuvre de politiques publiques adaptées: des politiques économiques de redistribution de la richesse, des politiques relatives aux mutations techniques de la société; des politiques urbaines et territoriales qui ne peuvent être conçues qu'à partir de démarches, d'analyses et de projets tous situés au-delà des échelles du local. Ma thèse est donc que ces trois scénarios nous invitent désormais à engager une réflexion top down en utilisant tout ce que nous a appris l'approche bottom up.

\section{Changement global}

Deux thématiques prioritaires se sont affirmées ces dernières années pour contrecarrer les conséquences de l'augmentation des températures sur la planète, qui affecte un grand nombre de processus « naturels »: la gestion de l'eau et les économies d'énergie, avec l'écho de production des énergies alternatives à l'énergie fossile.

Les expériences récentes auxquelles j'ai fait référence montrent que les problèmes de gestion de l'eau ne peuvent pas être résolus uniquement par de grands bassins de stockage qui viendraient garantir des réserves pour des régions entières, les mettant à l'abri des risques d'inondation et de manque d'eau pour leurs populations et leurs activités, agriculture comprise. II faut au contraire concevoir la présence de nombreux petits bassins dispersés et de petits réseaux très connexes, peu hiérarchisés, de canaux de collecte et de redistribution de l'eau. Des recherches récentes ont permis de lire à nouveau les caractéristiques de vastes territoires qui, pendant des siècles d'intervention hydraulique, ont été construits par l'eau en donnant " plus d'espace à l'eau » et en organisant une re-naturalisation des cours d'eau à tous les niveaux. Les réflexions sur la revégétalisation conduisent à la même conclusion: il ne s'agit pas tant de reboiser certaines parties de pays ou de continents que d'agir de manière diffuse et cohérente sur les réseaux hydrauliques, le tout en renaturalisant des secteurs entiers, en ménageant des corridors naturels et en restaurant des systèmes de végétation préexistants.

On arrive à la même conclusion quand on réfléchit aux alternatives à l'énergie fossile et aux principales stratégies en matière d'économies d'énergie. En effet, il ne convient pas tant de construire de grandes centrales pour capter et transformer l'énergie, notamment solaire et éolienne, que de promouvoir le développement d'un ensemble de petits (ou très petits) systèmes de production d'énergie autonomes tout en assurant la diffusion de multiples dispositifs (même minuscules) d'économie d'énergie.

Toutes ces perspectives d'action peuvent être réalisées plus facilement dans la ville diffuse que dans la ville consolidée et ses banlieues. Mais chacune d'elles suggère la mise en œuvre de politiques et la réalisation de projets à l'échelle de secteurs suffisamment vastes pouvant coïncider par exemple avec des bassins fluviaux 
et/ou des systèmes de bassins fluviaux. Chacune de ces perspectives requiert donc des études interdisciplinaires confiées à de nouvelles constellations de chercheurs et à de nouvelles compétences d'expertise.

\section{Urbanisation}

La population de la planète tend à se concentrer dans de grandes régions urbaines. Vu du sous-continent indien, du Sud-Est asiatique, de l'Amérique latine ou de la Chine, le continent européen ne compte que des agglomérations urbaines de taille moyenne ou petite. Même les grandes métropoles européennes ont des dimensions beaucoup plus modestes que celles qui se développent aujourd'hui dans d'autres parties de la planète. Avec une longue histoire et de fortes institutions, les villes de taille moyenne ou petite ont toujours opposé en Europe une fière résistance à l'hégémonie des grandes capitales. Les polémiques du XVII siècle à l'égard de la grande ville et les controverses du XIX $X^{e}$ siècle à l'égard de la métropole, lieu du luxe et de la consommation inutile, ne se sont jamais éteintes. Fière de son université ou de sa brasserie, de son couvent ou de son musée, de son théâtre ou de son hôpital, de ses rites ou de ses académies, des personnalités ou des événements qui au cours du temps l'ont illustrée, la petite ville s'est toujours située à un niveau plus élevé que celui de sa réelle dimension démographique et productive. Aujourd'hui encore, dans le contexte planétaire, l'importance politique et culturelle des villes et métropoles européennes n'est pas étroitement liée à leurs dimensions physiques et démographiques.

Les vastes déplacements de population dont l'urbanisation actuelle est issue ont de multiples origines, bien différentes toutefois de celles qui ont autrefois poussé les Européens de l'exode rural à s'établir dans des villes proches. Elles sont aussi différentes de celles qui avaient incité à l'émigration vers les villes du continent américain. Elles sont enfin différentes de celles qui expliquent les divers mouvements migratoires internes au continent européen après la Seconde Guerre mondiale.

Ce qui marque cependant le monde des villes contemporaines n'est pas tant l'absence d'inquiétude sur la croissance des agglomérations urbaines que le consensus qui l'accompagne, le fait d'y voir un rapide processus de modernisation socio-économique et politique, le fait d'y voir la formation de vastes marchés globaux grâce auxquels le monde serait de moins en moins inégalitaire. Or les grandes régions urbaines où se concentre la population de la planète sont bien éloignées de la ville consolidée et de ses banlieues. De façon apparemment désordonnée, des fragments d'urbanisation occupent souvent de très vastes territoires en créant généralement des problèmes environnementaux toujours plus difficiles, cela d'autant plus que s'y ajoutent désormais les problèmes de la pollution atmosphérique et de la pollution des sols progressivement imperméabilisés. La leçon de Los Angeles semble n'avoir rien appris.

\section{Riches et pauvres}

À l'échelle planétaire et dans la majorité des pays riches (notamment les plus riches), de nombreux observateurs enregistrent aujourd'hui un écart croissant entre richesse et pauvreté. De nombreux experts s'accordent aussi à reconnaître que les régions urbaines sont les lieux où ces différences deviennent plus visibles. 
Or la ville a été longtemps imaginée, dans les cultures occidentales, comme un lieu magique, comme le siège privilégié de toutes les innovations techniques et scientifiques, culturelles et institutionnelles, comme le creuset de toutes les formes de bio-politique (Cavalletti, $2005^{18}$ ). Elle a été imaginée comme un espace d'intégration sociale et culturelle permettant aux individus d'entrer en contact les uns avec les autres, de se connaître, d'apprendre les uns des autres et d'échanger éventuellement le meilleur de leur propre culture dans un processus d'hybridation continue, producteur de nouvelles identités, de nouveaux sujets et de nouvelles idées. Mais depuis toujours, et selon des modalités diverses, la ville a été également une machine de distinction et de séparation, de marginalisation et d'exclusion de groupes ethniques et religieux, d'activités et de professions, d'individus et d'ensembles d'individus, de riches et de pauvres. Dans la ville occidentale, les riches et les pauvres se sont toujours rencontrés et continuent à se rencontrer, mais ils sont aussi devenus, manifestement, plus distants.

Le riche n'est pas seulement la personne, la famille, le groupe qui dispose de revenus élevés ou d'un patrimoine imposant, le riche est aussi celui qui, pour employer les termes de Pierre Bourdieu $\left(1979^{19}\right)$, possède un patrimoine culturel consistant, c'est-à-dire un savoir ou un niveau professionnel élevé, un solide capital social, un réseau de relations (en particulier avec les "puissants ») qui lui confère un statut et souvent des revenus analogues à ceux des personnes dotées d'un capital économique élevé.

De même, le pauvre n'est pas seulement la personne, la famille ou le groupe qui dispose de revenus ou d'un patrimoine réduits, c'est aussi celui qui ne peut pas disposer comme les autres (même potentiellement) de la possibilité d'accéder à certains biens essentiels à sa survie, à certains droits de citoyenneté, à certaines fonctions d'assistance sociale sous leurs différentes formes.

Les deux groupes sociaux, celui des riches et celui des pauvres, ont ainsi une identité changeante et des contours incertains. Au fil du temps et dans les différentes régions de la planète, ils ont été perçus et définis de manière différente. Dans notre "société liquide" contemporaine (Bauman, 2003 ${ }^{20}$ ), les groupes sociaux sont habituellement assez articulés les uns aux autres. Mais ils ne sont pas clairement définis par leur position dans les processus de production, comme c'était schématiquement le cas naguère pour la bourgeoisie et la classe ouvrière. Ils ne sont pas non plus définis par leur position dans une hiérarchie de propriété et de juridiction territoriale consolidées, comme c'était le cas de l'aristocratie sous I'Ancien Régime. Leur identité (avec ses limites) s'exprime plus simplement par la géographie du pouvoir, qui est plus vague, plus mouvante, souvent contradictoire et conflictuelle. II faut dire surtout que riches et pauvres ne sont plus des catégories spéculaires. L'absence de ce qui définit la richesse ne contribue pas toujours à définir la pauvreté et, inversement, ce qui définit la pauvreté est souvent inadéquat pour définir la richesse. Entre ces deux situations existe une zone intermédiaire, un entre-deux indéfinissable qui joue souvent un rôle assez important d'occultation des situations extrêmes.

Dans les sociétés modernes démocratiques, l'ensemble des riches est en principe un ensemble ouvert:
18. Andrea Cavalletti, La città biopolitica. Mitologie della sicurezza, Milan, Bruno Mondadori, 2005.

19. Pierre Bourdieu, La distinction. Critique sociale du jugement, Paris, Les Éditions de Minuit, 1979
20. Zygmunt Bauman, Liquid Life, Cambridge, Polity Press, 2003 ; trad. fr. La vie liquide, Rodez/Nîmes, Le Rouergue/ Chambon, coll. "Les Incorrects », 2006. 

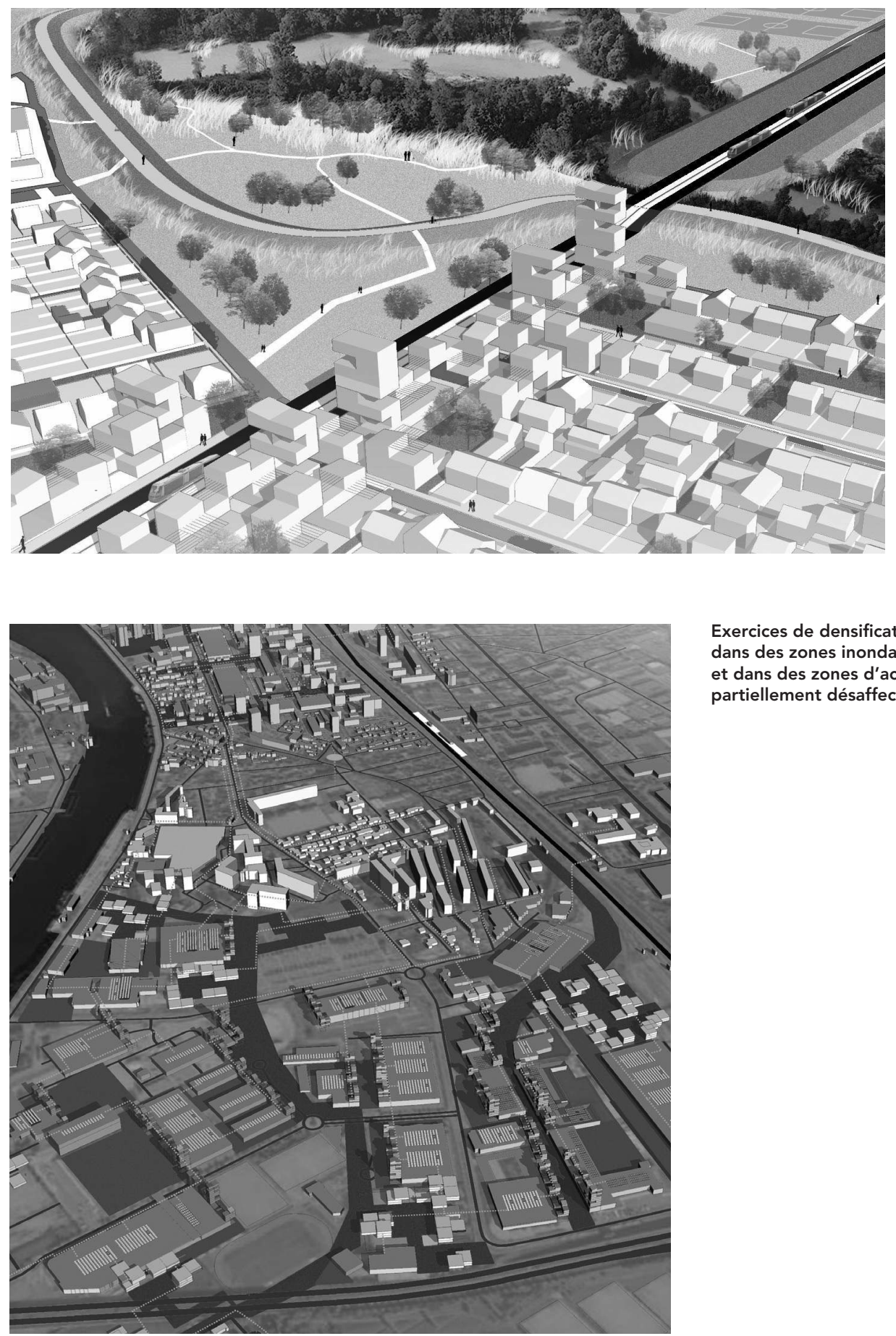

Exercices de densification dans des zones inondables et dans des zones d'activités partiellement désaffectées. 
196 Les Cahiers de la recherche architecturale et urbaine - n०24/25 $\quad$ La critique en temps et lieux

La partie nord

de la " traversée verte ".

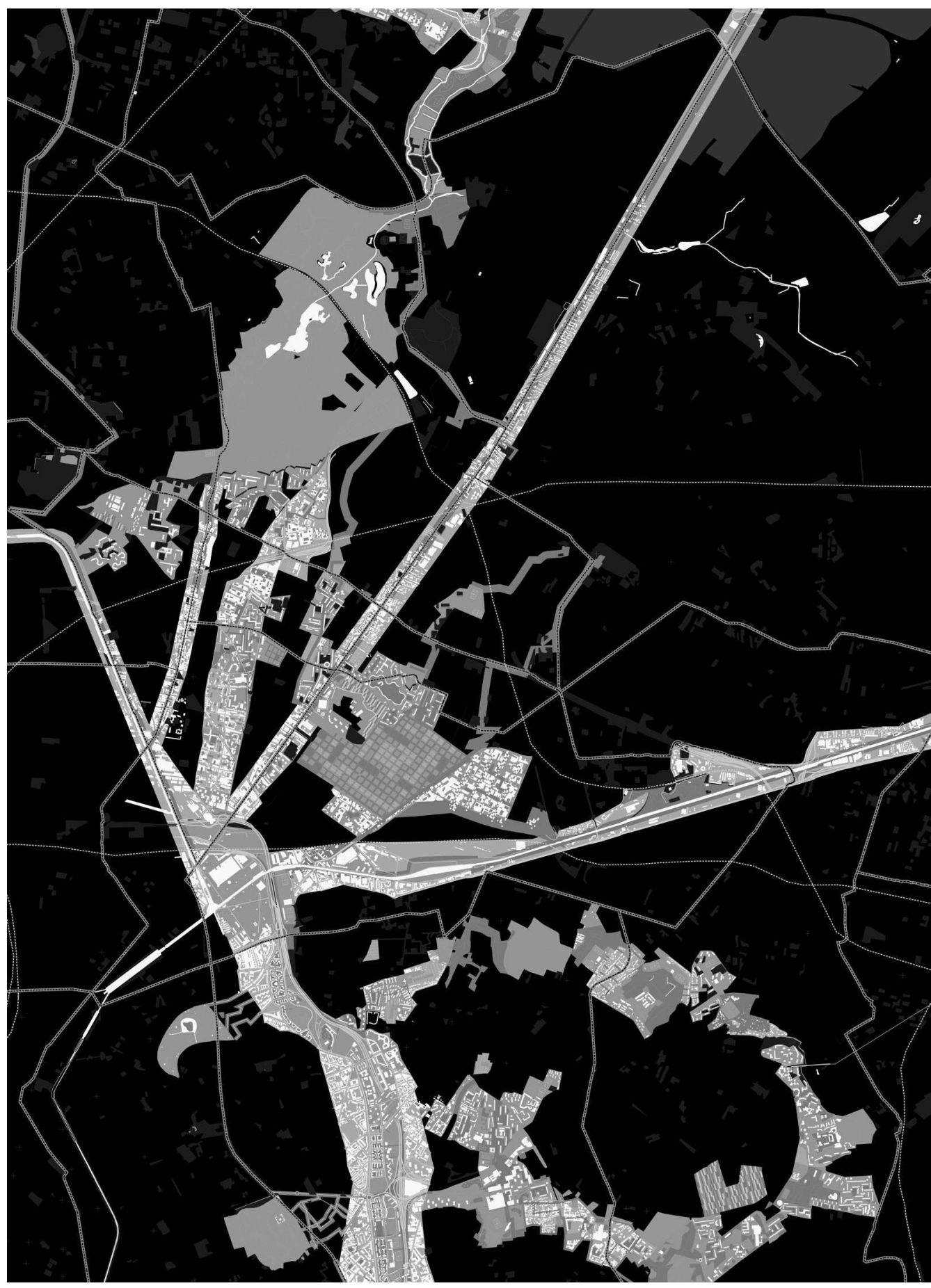


chacun peut espérer y entrer et tous devraient savoir que le risque d'en être expulsé est réel. Dès lors, il n'est pas étonnant que le groupe social des riches, comme tous les groupes sociaux dominants du passé qui entraient en conflit avec les autres au prix de la restriction de l'espace démocratique, cherche à faire valoir comme principe d'évolution et d'autodéfense un principe indirect de cooptation sélective. Autrement dit, les membres du groupe social des riches cherchent à utiliser des dispositifs qui sont parfois de nature spatiale (Flusty, $1997^{21}$ ) pour se définir des règles de comportements internes, pour contrarier l'arrivée des autres et pour donner une visibilité à ceux qui en font partie. Par certains aspects, la ville contemporaine paraît être le lieu d'expression de ces stratégies, de ces conflits et de ces représentations de leurs résultats provisoires.

Dans l'histoire plus récente de la ville occidentale, on peut identifier les différentes manières (en termes de tradition, de conceptualisation et de gouvernance) d'appréhender les problèmes $d^{\prime}$ inégalité entre les riches et les pauvres. À celles-ci correspondent des pratiques et des dispositifs souvent régis par des normes partagées. Naturellement, il peut sembler plus simple de les appréhender en examinant d'abord la nature des politiques économiques (notamment fiscales), la structure des institutions qui renforcent ou affaiblissent les droits à la citoyenneté, ou encore l'évolution des comportements citadins au sujet de la pauvreté et des moyens de l'alléger (Friedman, 2005 22 ). Mais on peut aussi partager l'opinion de Carl Schmitt selon laquelle « il n'existe pas d'idées politiques sans un espace auquel les référer, ni d'espaces ou de principes spatiaux auxquels ne correspondent des idées politiques »(Schmitt, 193923). Dans cet ordre d'idées, il y a matière à explorer un terrain plus difficile: celui des dispositifs spatiaux qui organisent la ville et le territoire tout en ayant d'importantes conséquences sur les relations d'intégration ou d'exclusion entre les riches et les pauvres.

Un exemple: la concentration dans les grandes villes de ceux qui depuis toujours incarnent le peuple, la foule et la masse, a toujours et partout inquiété, au moins autant que la maladie et la famine qui leur étaient souvent associées. C'est particulièrement vrai pour la concentration des misérables, des classes dangereuses ou plus simplement des pauvres. Comme la lèpre, la peste et la variole, cette concentration est à l'origine de peurs individuelles et collectives qui ont façonné les imaginaires. Comme la lèpre, la peste et la variole, elle a souvent suscité aussi la mise en œuvre de politiques spécifiques d'exclusion, d'éloignement ou d'internement (Foucault, 1975 ${ }^{24}$ ) qui conduisent forcément à rechercher un coupable: le « différent » et surtout l'« étranger » (Bauman, 2005 ${ }^{25}$ ).

Autre exemple: I'hétérogénéité et la fragmentation de la ville contemporaine plongent leurs racines dans la rupture progressive des systèmes de solidarité et dans l'émergence correspondante de systèmes d'intolérance. Ces systèmes peuvent être de nature sanitaire, religieuse, ethnique ou culturelle. Ils peuvent concerner des modes de vie ou des niveaux de vie différents, des habitudes de consommation ou des choix portant sur les caractéristiques de l'espace habitable. À chaque fois qu'ils se sont transformés, la configuration de la ville et du territoire a changé. En Occident et peut-être même partout ailleurs sur la planète, à chaque période de développement des
21. Steven Flusty, Building Paranoia: The Proliferation of Interdirectory Space and the Erosion of the Spatial Justice, in N. Ellin, E. J. Blakely (dir.), Architecture of Fear, New York, Princeton Architectural Press, 1997

22. Benjamin M. Friedman, The Moral Consequences of Economic Growth, New York, Vintage Books, 2005.
23. Carl Schmitt, Völkerrechtliche Grossraumordnung mit Interventionsverbot für raumfrende Mächte. Ein Beitrag zum Reichsbegriff im Völkerrecht, Berlin/Vienne, 1939 ; aujourd'hui in C. Schmitt, Staat, Grossraum, Nomos. Arbeiten aus den Jahren 1916-1969, Berlin, Duncker et Humblot, 1995.
24. Michel Foucault, Surveiller et punir. Naissance de la prison, Paris, Gallimard, 2005 [1975].

25. Zygmunt Bauman, Trust and Fear in the Cities, 2005 (éd. italienne, Fiducia e paura nella città, Milan, Bruno Mondadori, 2005). 
civilisations urbaines, toute l'histoire de la ville et du territoire pourrait être écrite à partir de ces compatibilités et incompatibilités réciproques: la ville bourgeoise et la ville ouvrière, la ville des autochtones et celle des immigrés, la ville des différentes ethnies, etc.

Troisième exemple: la peur qui traverse le monde actuel n'est pas un phénomène nouveau car il n'y a jamais eu, par le passé, de période dans laquelle on n'ait pas eu peur. La peur traverse toute notre histoire depuis la plus lointaine Antiquité (Delumeau, 1978 ${ }^{26}$ ): peur de l'agression de l'ennemi, des Infidèles, de la maladie et de la contagion; peur de la famine et de la faim; peur des calamités provoquées par une nature ingrate; peur suscitée parl'« autre » et par le « différent». La nature de ce qui fait peur change. Avec elle changent aussi la rhétorique de la sécurité et les dispositifs mis en œuvre pour combattre cette peur: choix de lieux inaccessibles bien défendus par des barrières naturelles; construction de remparts et de murs; dispositifs juridiques qui limitent l'entrée, la sortie ou le transit tout en définissant le statut spécifique de lieux et territoires déterminés. Le catalogue de ces dispositifs n'est pas infini ; tout au long de l'histoire, ils se sont perfectionnés en fonction des priorités qu'on leur a accordées. Surtout, ils ont été combinés et légitimés par une société de la communication pour laquelle les rhétoriques ont des conséquences matérielles souvent plus évidentes que les faits mêmes qu'elles cherchent à évoquer.

Mais dans la ville et le territoire, la peur n'est pas seule responsable des distinctions et séparations spatiales des individus et des groupes sociaux, des riches et des pauvres. Parmi les traditions scientifiques et professionnelles qui ont longtemps œuvré dans la même direction, les politiques publiques de la ville et du territoire occupent une place privilégiée. Nommer, localiser et définir, spécifier et délimiter, séparer et éloigner, lier et conjuguer, ouvrir et clôturer, donner des dimensions concrètes à chaque ensemble construit ou à chaque matériau urbain (par exemple à un ensemble d'édifices résidentiels ou d'équipements scolaires, à une rue ou à un jardin), tels sont les éléments constitutifs du projet de la ville et du territoire. Tels sont aussi (et en même temps) les principaux dispositifs de contrôle de la compatibilité et de l'incompatibilité, de la redistribution du bien-être et de la construction d'une idée partagée de la sécurité. II convient donc de définir les caractéristiques morphologiques et typologiques d'une partie de cette ville: prévoir de grands slabs plutôt que des maisons individuelles avec jardin; spécifier son niveau d'infrastructuration et d'équipements (combien d'espaces verts, de parkings, de jardins d'enfants, d'écoles et de centres sanitaires); les séparer ou les associer au centre-ville, à la ville existante ou à d'autres parties semblables; la doter ou non de liaisons faciles et rapides en transports en commun ; la situer sur les pentes des collines ou au fond des vallées (sous le vent, à proximité d'une zone industrielle ou donnant sur la mer) ou bien la séparer d'autres parties de la ville, différentes par leurs populations et leurs activités; disposer entre les zones urbanisées de vastes espaces ouverts ou des barrières infrastructurelles; entourer les diverses zones de ceintures vertes, de murs ou de clôtures.

Chacun de ces dispositifs a été détaillé dans les nombreux discours et écrits des architectes et des urbanistes, dans des déclarations ayant des prétentions de scientificité, dans des prises de position morales, dans 


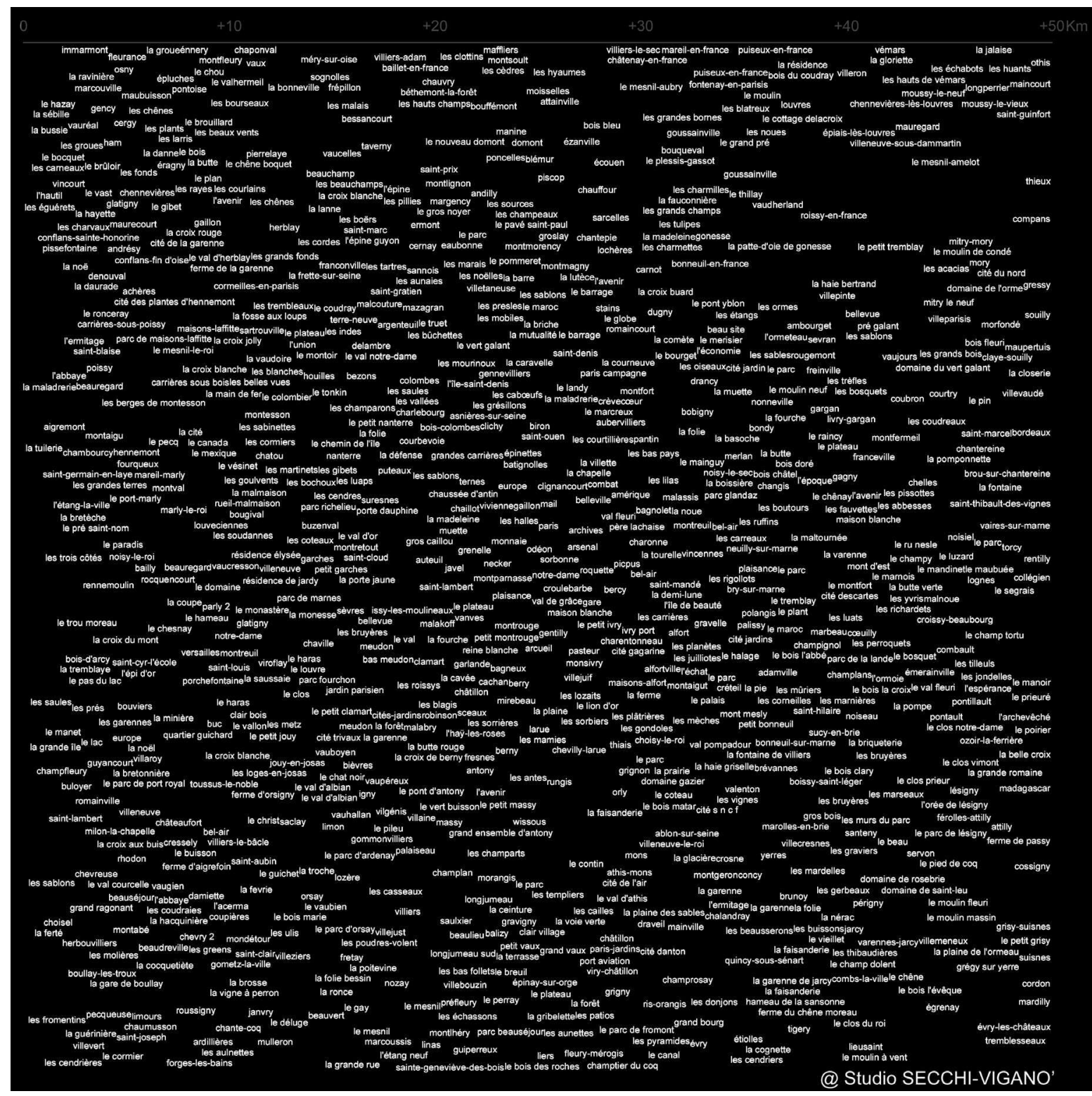

Carte des lieux nommés

(et pourtant significatifs) dans

le Grand Paris. 
des institutions spécifiques, des procédures administratives, des normes et des règlements, des concepts juridiques et, naturellement, dans les propositions et les réalisations de nombreux plans d'urbanisme et de nombreux projets d'architecture.

\section{Nous avons encore beaucoup à apprendre...}

Ces réflexions ont une conclusion très simple: il faut recommencer à élaborer des travaux de recherche et des explorations de projets à grande échelle en donnant la priorité à une démarche de research by design. Les thèmes proposés par les trois grands scénarios auxquels j'ai fait référence demandent des réponses cohérentes aux échelles les plus pertinentes. En effet, nous avons besoin d'explorer ces domaines complexes du projet en y introduisant le potentiel de ces thèmes de réflexion et en privilégiant une vision générale qui tienne compte de la singularité des lieux. En d'autres termes, après des décennies de recherches et de projets locaux caractérisés par une approche de type bottom up, il faut avoir le courage d'affirmer la valeur d'une approche théorique et expérimentale de type top down.

TRADUCTION DE L'ITALIEN PAR ANNE GRILLET-AUBERT

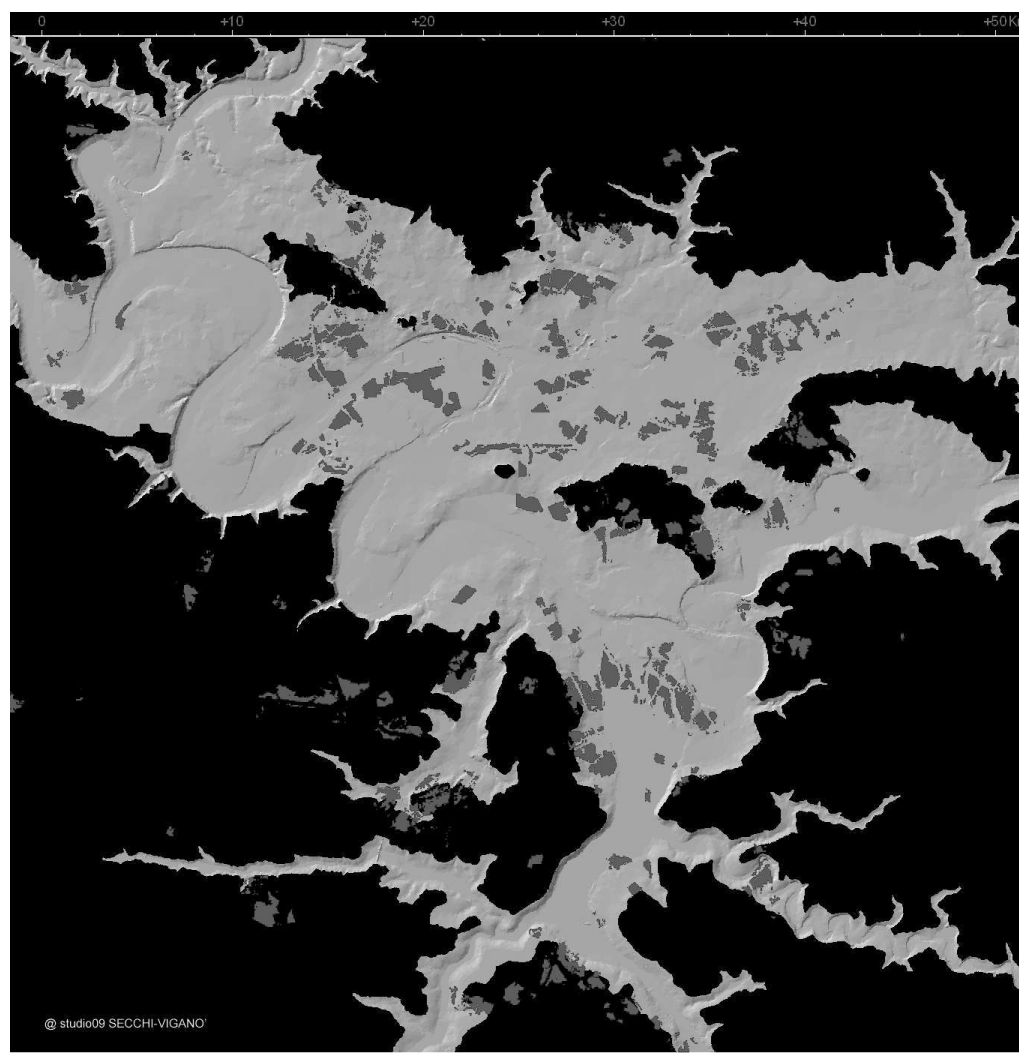

Détail de la « traversée verte ". 


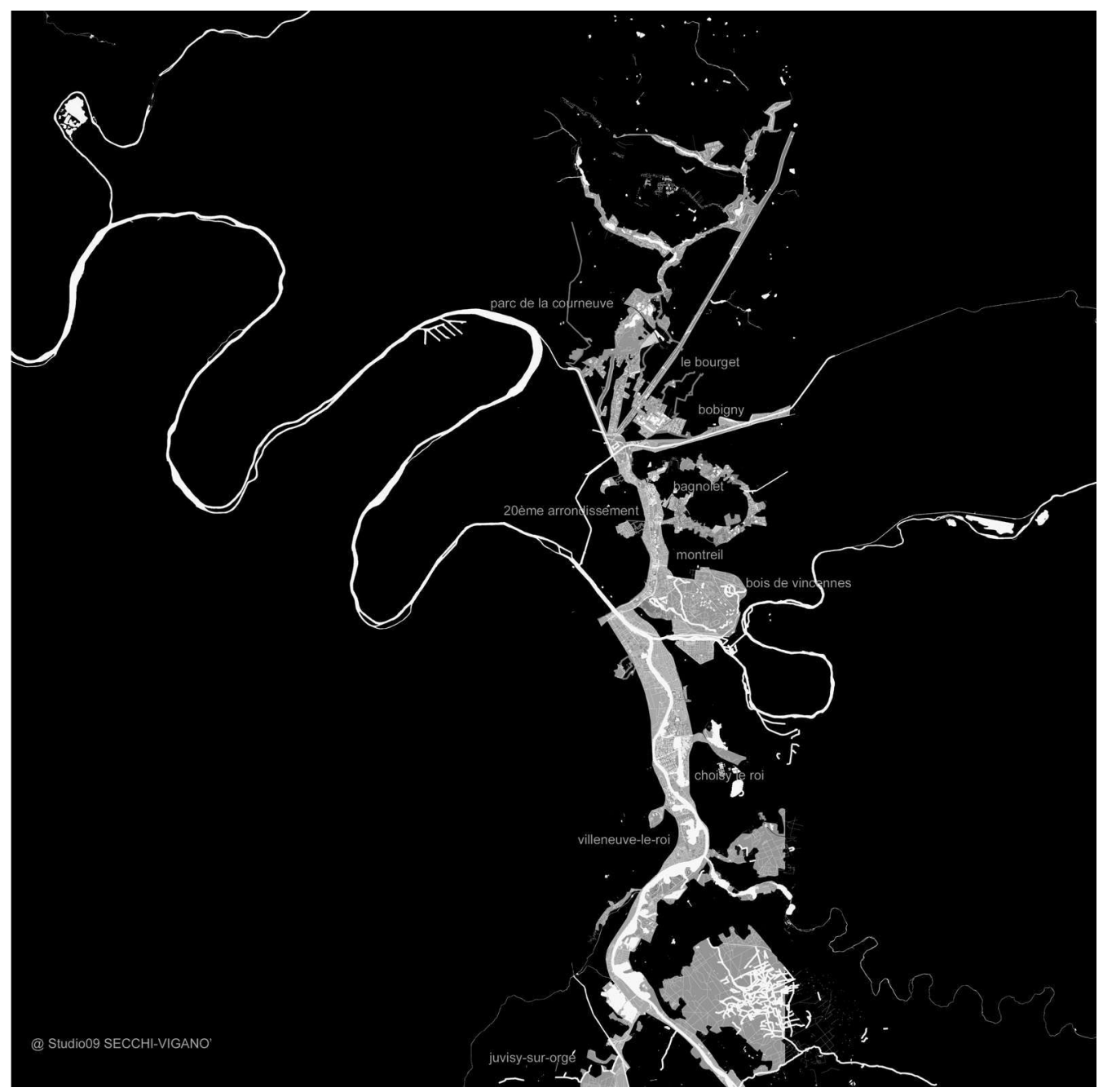

La «traversée verte ». 